\title{
On the Lagrangian Description and Uniqueness for the one- dimensional Pressureless Euler System
}

\author{
Mark David Suder \\ WVU, msuder@mix.wvu.edu
}

Follow this and additional works at: https://researchrepository.wvu.edu/etd

Part of the Analysis Commons

\section{Recommended Citation}

Suder, Mark David, "On the Lagrangian Description and Uniqueness for the one-dimensional Pressureless Euler System" (2021). Graduate Theses, Dissertations, and Problem Reports. 10184.

https://researchrepository.wvu.edu/etd/10184

This Dissertation is protected by copyright and/or related rights. It has been brought to you by the The Research Repository @ WVU with permission from the rights-holder(s). You are free to use this Dissertation in any way that is permitted by the copyright and related rights legislation that applies to your use. For other uses you must obtain permission from the rights-holder(s) directly, unless additional rights are indicated by a Creative Commons license in the record and/ or on the work itself. This Dissertation has been accepted for inclusion in WVU Graduate Theses, Dissertations, and Problem Reports collection by an authorized administrator of The Research Repository @ WVU.

For more information, please contact researchrepository@mail.wvu.edu. 
On the Lagrangian Description and Uniqueness for the one-dimensional Pressureless Euler System

\author{
Mark David Suder \\ Dissertation submitted to the \\ Eberly College of Arts and Sciences \\ at West Virginia University \\ in partial fulfillment of the requirements \\ for the degree of
}
Doctor of Philosophy
in
Mathematics
Adrian Tudorascu, Ph.D., Chair
Charis Tsikkou, Ph.D.
Harumi Hattori, Ph.D.
Jerzy Wojciechowski, Ph.D.
Tudor Stanescu, Ph.D.

\author{
Department of Mathematics
}

Morgantown, WV

2021

Keywords: Pressureless Euler, Sticky Particles System, Sticky Particles Flow Equation, Scalar Conservation Laws, Lagrangian Coordinates; MSC 2010:

35A02, 35C99, 35F50, 35Q70, 35R06

Copyright 2021 Mark David Suder 


\title{
ABSTRACT \\ On the Lagrangian Description and Uniqueness for the one-dimensional Pressureless Euler System
}

\author{
Mark David Suder
}

In this work we show that the one-dimensional pressureless Euler system admits a Lagragian characterization under fairly general initial conditions, extending recent results by Hynd [7]. Moreover, we show that if the initial velocity is right-continuous and bounded, then we have uniqueness of this Lagrangian solution (called Sticky Particles Flow, or SPF solution), which coincides with the Scalar Conservation Laws (or SCL) solution.

An important tool we employed in order to prove existence is a result by Gangbo et al. [5], which establishes a canonical (i.e. the flow is given by the optimal maps pushing the Lebesgue measure restricted to the unit interval forward to the measure-valued solutions) Lagrangian representation of an absolutely continuous flow. Besides the existence result for Lagrangian solutions, which generalizes a recent result by Hynd [7], we obtain uniqueness of said solutions as our main contribution to the field.

The uniqueness issue is a long-standing one, with only partial results available. Extra, entropy-like conditions are necessary to single out a solution and such conditions are complicated by the fact that the generic space for existence is the Wasserstein space of probability measures. This means that the Oleinik entropy condition, for example, should naturally be imposed almost everywhere with respect to the measure-valued solution; however, the uniqueness literature uses "everywhere" conditions. These are delicate to obtain because generically the velocity of the flow is a priori well-defined almost everywhere with respect to the measurevalued solution. In this thesis we employ a meticulous extension procedure for the velocity of the flow, which produces the everywhere Oleinik condition as a consequence of the usual, a.e. condition. 
To my wife Margie, for believing in me, even if I make this stuff up;

And to my advisor Adrian, for showing me the path to some beautiful new mathematics. 


\section{Contents}

1 Pressureless Euler System 1

1.1 Physical System . . . . . . . . . . . . . . . . . . . . . . . 1

1.2 Overview and Main Results . . . . . . . . . . . . . . . . . . . . . 3

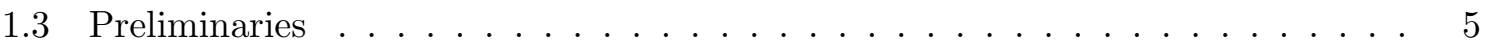

1.4 Previous Results . . . . . . . . . . . . . . . . . 7

2 Measure Theory and Optimal Transport $\quad 8$

2.1 Measure Theory . . . . . . . . . . . . . . . . 8

2.2 Optimal Transport . . . . . . . . . . . . . . . . . . . 17

3 Lagrangian Description of Pressureless Euler System Solutions $\quad 19$

3.1 SPF solutions to $(\mathrm{PE}-\mathrm{IC}) \ldots \ldots \ldots \ldots \ldots \ldots$

3.2 SCL solutions to $(\mathrm{PE}-\mathrm{IC}) \ldots \ldots \ldots \ldots \ldots \ldots \ldots$

3.3 Equivalence of SPF and SCL Solutions . . . . . . . . . . . . . 26

3.3 .1 Preliminary Lemmas . . . . . . . . . . . . . . . . 27

3.3 .2 From $($ Oleinik) to $($ e-Oleinik $) \ldots \ldots \ldots \ldots \ldots$

3.3.3 SPF solution satisfies Corollary 3.3 .1 assumptions . . . . . . . . . . . 35

3.3.4 SCL solution satisfies Corollary 3.3 .1 assumptions _ . . . . . . . . . . . 36

3.4 Existence of an SPF solution for general initial data . . . . . . . . . . . . 38

3.4 .1 More General $v_{0} \ldots \ldots \ldots \ldots \ldots$

4 Open Problems $\quad 42$

4.1 Boundary conditions . . . . . . . . . . . . . . . . . 42

4.2 Unique Eulerian Solution . . . . . . . . . . . . . . . . . . . 44

5 Appendices $\quad 45$ 


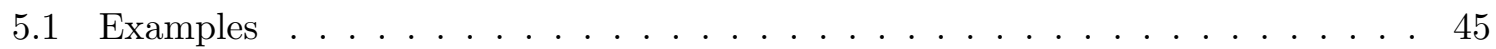

5.2 Borel Sets and Maps . . . . . . . . . . . . . . . . . . . . . . 46 


\section{Chapter 1}

\section{Pressureless Euler System}

\subsection{Physical System}

The pressureless Euler system is a system of partial differential equations that represent conservation laws. The first equation represents conservation of mass and is derived as follows. Suppose we have a fluid flow as shown in the diagram below.

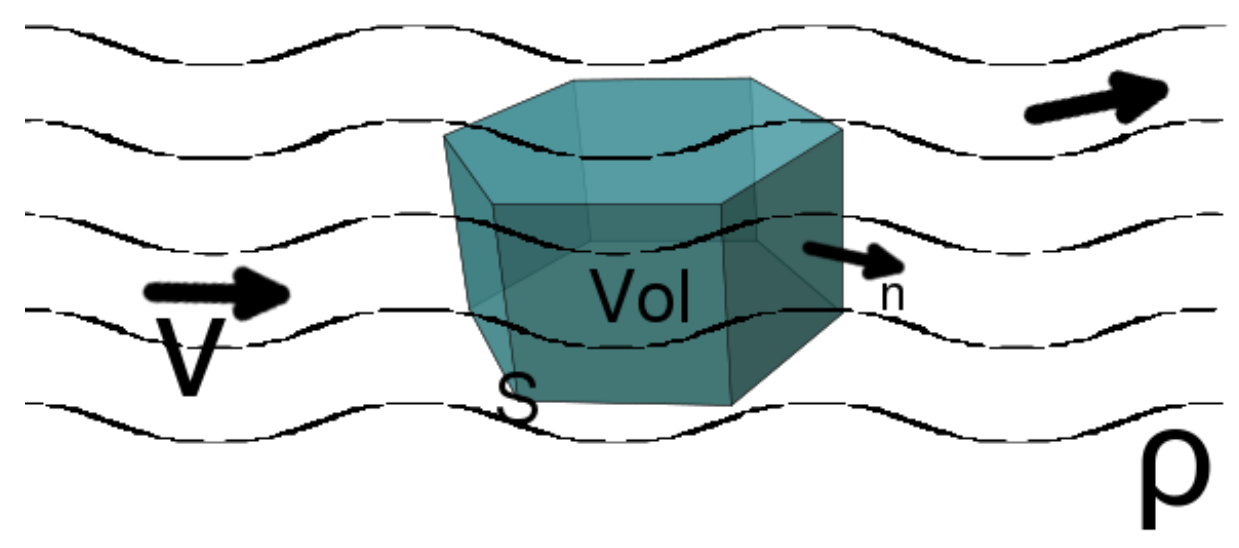

Figure 1.1: Conservation Equations

Then $\rho$ represents the fluid mass density at each point and $v$ represents the velocity of the fluid flow at each point.

Note that the total mass in $\mathrm{Vol}$ is given by:

$$
\iiint_{V o l}(\rho) d V o l
$$

Based on this and assuming smooth enough conditions, the rate of change of mass in $\mathrm{Vol}$ over time is given by: 


$$
\partial_{t} \iiint_{V o l}(\rho) d V o l=\iiint_{V o l}\left(\partial_{t} \rho\right) d V o l
$$

In addition, the mass flowing out of $V o l$ over time, i.e. the mass flowing through the surface $S$ of $V o l$ over time, is given by:

$$
-\iint_{S}((\rho v) \cdot \hat{n}) d S
$$

By Gauss' divergence theorem, we have:

$$
\iint_{S}(\rho v \cdot \hat{n}) d S=\iiint_{V o l}(\nabla \cdot \rho v) d V o l
$$

We then equate the rate of mass changes to get:

$$
-\iiint_{V o l}(\nabla \cdot(\rho v)) d V o l=\iiint_{V o l}\left(\partial_{t} \rho\right) d V o l
$$

Rearranging, we get:

$$
\iiint_{V o l}\left(\partial_{t} \rho+\nabla \cdot(\rho v)\right) d V o l=0
$$

Since this must hold true for any small volume $V$ with surface $S$, this results in the conservation of mass equation:

$$
\partial_{t} \rho+\nabla \cdot(\rho v)=0
$$

Referring again to the figure, we can now balance the forces present for the volume $V$. Given an external force $F$, the net external momentum is given by:

$$
\iiint_{V o l} \rho F d V o l
$$

The total momentum within $V o l$ is given by:

$$
\iiint_{V o l}(\rho v) d V o l
$$

Thus the rate of change of total momentum within $V o l$ is:

$$
\partial_{t} \iiint_{V o l}(\rho v) d V o l
$$

In addition, the rate of change of momentum across the boundary $S$ of $\mathrm{Vol}$ is given by: 


$$
\iint_{S} \rho v(v \cdot \hat{n}) d S+\iint_{S} p \hat{n} d S .
$$

In the above, $p$ is the pressure exerted on the boundary. We will assume the pressure is zero (pressureless Euler equations) and thus applying Gauss' divergence theorem again, the rate of change of momentum across the boundary will be:

$$
\iiint_{V o l} \nabla \cdot(\rho v \otimes v) d V o l
$$

Combining these change in momentum equations, we get:

$$
\partial_{t} \iiint_{V o l}(\rho v) d V o l+\iiint_{V o l} \nabla \cdot(\rho v \otimes v) d V o l=\iiint_{V o l} \rho F d V o l .
$$

We will assume the external force $F$ is zero and the functions are sufficiently smooth, resulting in:

$$
\iiint_{V o l} \partial_{t}(\rho v)+\nabla \cdot(\rho v \otimes v) d V o l=0
$$

Finally, since this must hold true for any small volume $V$ with surface $S$, this results in the conservation of momentum equation:

$$
\partial_{t}(\rho v)+\nabla \cdot(\rho v \otimes v)=0
$$

\subsection{Overview and Main Results}

The general Pressureless Euler Equations in one spatial dimension are the following:

$$
\left\{\begin{array}{r}
\partial_{t} \rho+\partial_{x}(\rho v)=0 \\
\partial_{t}(\rho v)+\partial_{x}\left(\rho v^{2}\right)=0
\end{array}\right.
$$

This is a pair of functional equations in the unknowns $\rho$ and $v$, where $\rho$ represents the mass density of the fluid at a particular location and time and $v$ represents the velocity of the fluid at a particular location and time. Several questions immediately arise. What are the allowable solution spaces for $\rho$ and $v$ ? In the present study, we will restrict the space of allowable initial mass distributions to probability measures with finite second moment. It should be noted that this is not an unnatural space, since in practical applications, the mass of the entire system is finite and so, without loss of generality, can be normalized to unity. The finite second moment condition is also natural, as this only quantifies the fact that these mass distributions do not escape at infinity; moreover, prior works obtained existence of solutions by studying the system in the context of the Wasserstein space. We will also restrict the space of allowable initial velocities to functions that are square integrable with respect to the initial mass density measure. 
So with this backdrop, we will study the one (spatial) dimensional PE system with initial conditions $\rho_{0}$ in the space of probability measures with finite second moment, i.e. $\rho_{0} \in \mathcal{P}_{2}(\mathbb{R})$, and $v_{0}$ in the space of square integrable functions with respect to $\rho_{0}$ :

$$
\left.\rho\right|_{t=0}=\rho_{0},\left.v \rho\right|_{t=0}=v_{0} \rho_{0} .
$$

We shall use (PE-IC) to denote the IVP consisting of (PE) augmented by the initial conditions (IC).

In this dissertation we shall present two main contributions; first we show that Lagrangian solutions (we call them Sticky Particle Flow solutions, or SPF solutions) as in [7] satisfy the strong initial continuity of the energy, which, together with the Oleinik condition, is a necessary ingredient for our uniqueness result. The latter will be obtained by means of explicitly linking the SPF solutions and the Scalar Conservation Laws solutions (SCL solutions; see [2] and [9]) to the distributional solutions to a related problem whose well-posedness was shown in [6]. More precisely, we show (Theorem 3.3.2) that if $v_{0}$ is right-continuous and bounded on the real line, then any SPF solution must coincide with the (unique) SCL solution. Beside the strong initial continuity of the energy condition (SICE), a stronger version of the Oleinik condition is necessary for this connection (with the [6] solutions) to work; we meticulously prove that the velocity $v$ admits a Borel representative $\hat{v}($ i.e. $\hat{v}(t, \cdot)=v(t, \cdot), \rho(t, \cdot)$-a.e. for all $t$ ) which satisfies a stronger version of the Oleinik condition, which we dub (e-Oleinik) (everywhere Oleinik), in the sense that the pertinent inequality is required to hold everywhere (and not just $\rho(t, \cdot)$-a.e.). This is reminiscent of the work by Bouchut and James [1], where a similar issue (of having a velocity defined everywhere) was encountered; note also that the uniqueness result (for duality solutions, as defined there) in their paper only holds for non-atomic initial distributions. Results on uniqueness of solutions to (PE-IC) are few and far between (and each of them [1], [6], [10] involves notions of solutions which are more particular than simply distributional solutions or Lagrangian/SPF distributional solutions), so we believe ours to be an important contribution to this area of research.

Secondly, we show (Theorem 3.4.1) that SPF solutions exist for any initial velocity $v_{0} \in$ $L^{2}\left(\rho_{0}\right)$, thus eliminating the restriction from [7] that $v_{0}$ be absolutely continuous on $\mathbb{R}$. This is achieved by approximating the initial velocity by continuous and bounded functions and using the uniqueness result mentioned above in order to identify the SPF and SCL solutions for the approximating initial data; this identification plays a crucial role here, since it is known [9], [10] that the SCL solutions are stable with respect to the initial conditions.

We conclude this introduction with some preliminaries and then some previous results. Chapter 2 provides some measure theory and optimal transport background. The main results follow in Chapter 3. In Section 3.1 we show that SPF solutions satisfy the strong initial continuity of the energy. In Section 3.2 we gather some facts about SCL solutions, whereas in Section 3.3 we prove that for right-continuous and bounded initial velocities any SPF solution is an SCL solution (which, due to the uniqueness of the latter, implies uniqueness of the SPF solution under these assumptions on the initial velocity). Furthermore, since SCL solutions are stable with respect to initial data, in Section 3.4 we conclude that the SPF/SCL solutions corresponding to $\rho_{0} \in \mathcal{P}_{2}(\mathbb{R})$ and initial velocities $v_{0, n} \in C_{c}(\mathbb{R})$ (which converge in $L^{2}\left(\rho_{0}\right)$ to $\left.v_{0} \in L^{2}\left(\rho_{0}\right)\right)$ converge to an SPF solution for (PE-IC). In chapter 4 we describe several open problems. Finally, in order to illustrate the necessity of (Oleinik) and (SICE), in Section 5.1 we produce an example of three distinct distributional solutions to the same (PE-IC), only 
one of which satisfies both conditions, while each of the other two solutions satisfies exactly one of the two conditions. We conclude with Section 5.2, where we prove the measurability of various sets and maps used throughout some of the constructive proofs in Chapter 3 .

\subsection{Preliminaries}

Several tools will be used in this dissertation, which are described below. Throughout this manuscript we denote by $t \in[0, \infty)$ the time variable and by $y \in \mathbb{R}$ the spatial variable; we reserve $x$ for the real numbers in the open interval $(0,1)$. This way, for any Borel probability measure $\rho$ on $\mathbb{R}$ we have the right-continuous optimal map $N=N(x)$ which pushes the Lebesgue measure restricted to $(0,1)$ forward to $\rho$, while its generalized inverse is the rightcontinuous cumulative distribution function of $\rho$, denoted by $M=M(y)$.

Notation 1.3.1. The set of Radon measures on $\mathbb{R}$ will be denoted by $M(\mathbb{R})$.

Notation 1.3.2. The set of Borel probability measures on $\mathbb{R}$ will be denoted by $\mathcal{P}(\mathbb{R})$.

Notation 1.3.3. The set of Borel probability measures on $\mathbb{R}$ with finite second moment, i.e. those measures $\rho \in \mathcal{P}(\mathbb{R})$ such that

$$
\int_{\mathbb{R}} y^{2} \rho(d y)<\infty
$$

will be denoted by $\mathcal{P}_{2}(\mathbb{R})$.

Definition 1.3.1. A sequence $\left\{\rho_{n}\right\}_{n \in \mathbb{N}} \subset \mathcal{P}(\mathbb{R})$ converges narrowly to $\rho$ if

$$
\int_{\mathbb{R}} g d \rho_{n} \underset{n \rightarrow \infty}{\longrightarrow} \int_{\mathbb{R}} g d \rho
$$

for each $g \in C_{b}(\mathbb{R})$, where $C_{b}(\mathbb{R})$ is the set of continuous, bounded functions on $\mathbb{R}$.

Definition 1.3.2. Given $(P E-I C)$ with $\rho_{0} \in \mathcal{P}_{2}(\mathbb{R}), v_{0} \in L^{2}\left(\rho_{0}\right)$, a weak solution to this IVP is a pair $(\rho, v)$ consisting of a narrowly continuous $\rho:[0, \infty) \rightarrow \mathcal{P}(\mathbb{R})$ and a Borel map $v:[0, \infty) \times \mathbb{R} \rightarrow \mathbb{R}$ for which

1. For each $T>0$,

$$
\int_{0}^{T} \int_{\mathbb{R}} v^{2}(t, y) \rho(t, d y) d t<\infty .
$$

2. For each $\phi \in C_{c}^{\infty}(\mathbb{R} \times[0, \infty))$,

$$
\int_{0}^{\infty} \int_{\mathbb{R}}\left(\partial_{t} \phi+v \partial_{y} \phi\right)(t, y) \rho(t, d y) d t+\int_{\mathbb{R}} \phi(0, y) \rho_{0}(d y)=0 .
$$

3. For each $\phi \in C_{c}^{\infty}(\mathbb{R} \times[0, \infty))$,

$$
\int_{0}^{\infty} \int_{\mathbb{R}}\left(v \partial_{t} \phi+v^{2} \partial_{y} \phi\right) \rho(t, d y) d t+\int_{\mathbb{R}} \phi(0, y) v_{0}(y) \rho_{0}(d y)=0
$$


Definition 1.3.3. Given $\rho:[0, \infty) \rightarrow \mathcal{P}(\mathbb{R})$ and $v:[0, \infty) \rightarrow L^{2}\left(\rho_{t}\right)$, we say $(\rho, v)$ satisfies the Oleinik condition if

$$
\frac{v\left(t, y_{2}\right)-v\left(t, y_{1}\right)}{y_{2}-y_{1}} \leq \frac{1}{t} \text { for all } t>0 \text { and for } \rho(t, \cdot) \text {-a.e. } y_{1}<y_{2} \text {. }
$$

In what follows, we occasionally use (in order to unburden notation) the notation $\rho_{t}$ for $\rho(t, \cdot)$ and $v_{t}$ for $v(t, \cdot)$.

Definition 1.3.4. Given $\rho_{0} \in \mathcal{P}_{2}(\mathbb{R}), v_{0} \in L^{2}\left(\rho_{0}\right)$ and $\rho:[0, \infty) \rightarrow \mathcal{P}(\mathbb{R}), v:[0, \infty) \rightarrow L^{2}\left(\rho_{t}\right)$, $(\rho, v)$ satisfies the Strong Initial Continuity of Energy condition $(S I C E)$ if $\forall \phi \in C_{b}(\mathbb{R})$ :

$$
\int_{\mathbb{R}} v_{t}^{2} \phi d \rho_{t} \underset{t \rightarrow 0^{+}}{\longrightarrow} \int_{\mathbb{R}} v_{0}^{2} \phi d \rho_{0} .
$$

Definition 1.3.5. Given $\rho_{0} \in \mathcal{P}_{2}(\mathbb{R})$, $v_{0} \in L^{2}\left(\rho_{0}\right)$, and a Borel map $X: \mathbb{R} \rightarrow \mathbb{R}$, the conditional expectation of $v_{0}$ with respect to $\rho_{0}$ given $X$ is $f \circ X$, where $f \in L^{2}\left(X_{\#} \rho_{0}\right)$ is unique (guaranteed by the Riesz-Fréchet Representation Theorem on the Hilbert space $L^{2}\left(X_{\#} \rho_{0}\right)$ ) such that:

$$
\int_{\mathbb{R}} f(X(y)) \zeta(X(y)) \rho_{0}(d y)=\int_{\mathbb{R}} v_{0}(y) \zeta(X(y)) \rho_{0}(d y)
$$

for all $\zeta \in L^{2}\left(X_{\#} \rho_{0}\right)$.

The conditional expectation of $v_{0}$ with respect to $\rho_{0}$ given $X$ is denoted by $\mathbb{E}_{\rho_{0}}\left[v_{0} \mid X\right]$.

SPF solutions: In this dissertation, we make use of the Sticky Particles Flow Equation with Initial Condition formulation as in [7]

$$
\left\{\begin{array}{r}
\dot{X}(t)=\mathbb{E}_{\rho_{0}}\left[v_{0} \mid X(t)\right], \text { a.e. } t \geq 0 \\
X(0)=\operatorname{Id}_{\mathbb{R}} .
\end{array}\right.
$$

If $v_{0}$ is absolutely continuous, it is proved in [7] that (SPF-IC) admits a solution $X$ which is jointly Borel, absolutely continuous as a map $X:[0, \infty) \rightarrow L^{2}\left(\rho_{0}\right)$, and that there exists a Borel map $v:[0, \infty) \times \mathbb{R} \rightarrow \mathbb{R}$ such that

$$
\mathbb{E}_{\rho_{0}}\left[v_{0} \mid X(t)\right]=v(t, X(t, \cdot)) .
$$

Furthermore, if $\rho(t, \cdot)=X(t, \cdot)_{\#} \rho_{0}$, then $(\rho, v)$ is a distributional solution to (PE-IC).

SCL solutions: We also make use of Scalar Conservation Laws solutions (SCL solutions) to $(\mathrm{PE}-\mathrm{IC})$; for $\rho_{0} \in \mathcal{P}_{2}(\mathbb{R})$ and $v_{0} \in L^{2}\left(\rho_{0}\right)$ we consider

$$
\left\{\begin{array}{r}
\partial_{t} M+\partial_{y}[F(M)]=0 \\
M(0, \cdot)=M_{0},
\end{array}\right.
$$

where we make the following definitions:

1. $N_{0}$ is the optimal map such that $\left.N_{0 \#} \mathcal{L}^{1}\right|_{(0,1)}=\rho_{0}$.

2. $F$ is defined by $F(x)=\int_{0}^{x} v_{0} \circ N_{0}(s) d s$ for $x \in[0,1]$.

3. $M_{0}$ is defined by $M_{0}(y)=\rho_{0}((-\infty, y])$.

It is shown in [9], [10] that the entropy solution to (SCL-IC) produces a solution (SCL solution) to $(\mathrm{PE}-\mathrm{IC})$ via $\rho(t, \cdot)=\partial_{y} M(t, \cdot)$ and $v(t, \cdot) \rho(t, \cdot)=\partial_{y}[F(M(t, \cdot)]$. 


\subsection{Previous Results}

Zeldovich [14] introduced the Sticky Particles model in order to provide a raw description of the formation of large scale structures in the universe. The Sticky Particles model can be briefly described as follows. If $m_{i}, i=1, \ldots, n$ is a discrete system of masses initially located at $-\infty<x_{1}<\ldots<x_{n}<+\infty$ and moving with initial velocities $v_{i}, i=1, \ldots, n$, then one makes the assumption that the velocities remain constant while there is no collision. At the collision of a group of particles, the particles stick together and the initial velocity of the newly formed particle is given by the conservation of momentum. It turns out the evolution of this system is described by (PE-IC). In many of the works on existence of solutions [2], [3], [9], [10], [7], etc. the initial distribution is approximated by averages of Dirac masses and the ensuing Sticky Particles system is used to approximate solutions to (PE-IC).

The pressureless Euler system has been studied by different techniques in [2], [1], [7], [8], [9], [10], [3]. These techniques include the Sticky Particles model, description of the problem by an alternative scalar conservation law problem, and a semigroup approach. At the heart of our approach lies the identification (under appropriate initial conditions) of the Lagrangian solutions cf. Hynd [7] with the Scalar Conservation Laws solutions [2], [9], etc. We would also like to acknowledge the contribution in [8], where the solution is constructed by a Sticky Particles semigroup approach and a similar Lagrangian representation of the solution is obtained, albeit in a slightly weaker sense. The results in this dissertation build on a number of previous results for the one-dimensional pressureless Euler system.

In [7] a sticky particles approach is used. This approach asserts conservation of mass and momentum of a collection of particles that interact only via inelastic conditions. This work shows that for $\rho_{0} \in \mathcal{P}_{2}$ and absolutely continuous $v_{0}$, the sticky particles approach leads to existence of a Lagrangian solution of the one-dimensional pressureless Euler system.

In [2] and [9] a scalar conservation law approach is used. These works show that for $\rho_{0} \in \mathcal{P}_{2}$ and continuous $v_{0}$ of at most quadratic growth and finite energy that the scalar conservation law approach yields existence of a solution to the one-dimensional pressureless Euler system; they also obtain time regularity of the solution for the pressureless Euler system and that the velocity satisfies the Oleinik entropy condition.

Finally, in [8] explicit estimates of the solution in terms of the initial mass and momentum are obtained. This is done by constructing an evolution semigroup in a measure-theoretic phase space, allowing mass distributions in $\mathcal{P}_{2}(\mathbb{R})$ and velocity fields in $L^{2}$. 


\section{Chapter 2}

\section{Measure Theory and Optimal Transport}

\subsection{Measure Theory}

Since this dissertation depends heavily on measure theory, we recall here a number of standard definitions and results from measure theory. These can be found in any standard text on measure theory [11].

Notation 2.1.1. The set of natural numbers, $1,2, \ldots$ will be denoted by $\mathbb{N}$.

Definition 2.1.1. Given a set $X$, a collection of subsets $\Sigma$ is a sigma algebra of $X$ if

1. $X \in \Sigma$,

2. for all $Y \in \Sigma, X \backslash Y \in \Sigma$,

3. if $Y_{i} \in \Sigma$ for $i \in \mathbb{N}$, then $\bigcup_{i \in \mathbb{N}} Y_{i} \in \Sigma$.

Definition 2.1.2. The pair $(X, \Sigma)$ where $\Sigma$ is a sigma algebra of $X$ is called a measurable space.

Definition 2.1.3. Given a measurable space $(X, \Sigma)$, a function $\mu: \Sigma \rightarrow[0, \infty)$ is called a measure on $X$ if

1. for all $Y \in \Sigma, \mu(Y) \geq 0$,

2. $\mu(\emptyset)=0$,

3. if $\left\{\Sigma_{i}\right\}_{i=1}^{\infty}$ is a collection of disjoint subsets of $\Sigma$, then $\mu\left(\cup_{i=1}^{\infty} \Sigma_{i}\right)=\sum_{i=1}^{\infty} \mu\left(\Sigma_{i}\right)$.

Theorem 2.1.1. Suppose $(X, \Sigma)$ is a measurable space with measure $\mu$. Then

1. $X \in \Sigma$, 
2. if $Y_{i} \in \Sigma$ for $i \in \mathbb{N}$, then $\bigcap_{i \in \mathbb{N}} Y_{i} \in \Sigma$,

3. if $Y, Z \in \Sigma$ then $Y \backslash Z \in \Sigma$.

Definition 2.1.4. If $X$ is a measurable space, $Y$ is a topological space and $f: X \rightarrow Y$. Then $f$ is measurable if for every open set $V \in Y, f^{-1}(V)$ is measurable.

Theorem 2.1.2. Let $Y, Z$ be topological spaces, $X$ be a measurable space, $f: X \rightarrow Y$ be measurable, and $g: Y \rightarrow Z$ be continuous. Then $h=g \circ f$ is measurable.

Theorem 2.1.3. If $\mathcal{M}$ is a collection of subsets of $X$, then there exists a smallest sigma algebra $\Sigma$ containing $\mathcal{M}$.

Definition 2.1.5. Let $X$ be a topological space. There exists a smallest sigma algebra $\Sigma$ such that every open set of $X$ belongs to $\Sigma$. The elements of $\Sigma$ are called the Borel sets of $X$.

Definition 2.1.6. If $X$ is a measurable space with measure $\mu$ and $\mu(X)=1$ then $\mu$ is called $a$ probability measure.

Definition 2.1.7. If $X$ is a topological space, $\Sigma$ the sigma algebra of Borel sets of $X$ and $\mu: \Sigma \rightarrow[0, \infty]$ a measure on $X$, then the measure $\mu$ is called a Borel measure.

Definition 2.1.8. If $X$ is a topological, measure space with measure $\mu$ and $\mu(C)$ is finite for every compact subset of $X$, then $\mu$ is a Radon measure.

In addition to measure theory, this dissertation depends on optimal transport theory, which describes the transport of one measure to another. The following provides some standard definitions and results from optimal transport theory.

Definition 2.1.9. Given $\mu \in \mathcal{P}(\mathbb{R})$ and a Borel map $X: \mathbb{R} \rightarrow \mathbb{R}$, the push forward $\nu$ of $\mu$ by $X$, denoted $\nu=X_{\#} \mu$ is a measure $\nu \in \mathcal{P}(\mathbb{R})$ defined as $\nu(B)=\mu\left(X^{-1}(B)\right)$ for all Borel subsets $B$ of $\mathbb{R}$.

A useful, fundamental result from measure theory is the following:

Proposition 2.1.1. Given $\mu, \nu \in \mathcal{P}(\mathbb{R})$ and a Borel map $X: \mathbb{R} \rightarrow \mathbb{R}$,

$$
\nu=X_{\#} \mu \Longleftrightarrow \int_{\mathbb{R}} \phi(X(y)) \mu(d y)=\int_{\mathbb{R}} \phi(y) \nu(d y), \forall \phi \in C_{b}(\mathbb{R}) .
$$

\section{Recall the following:}

1. Definition/Notation: $S_{\#} \mu=\nu(S$ push forward $\mu$ equals $\nu)$ if $\forall B \subset Y$ with $B$ a Borel subset, $\mu\left(S^{-1}(B)\right)=\nu(B)$.

2. Notation: $C_{b}(Y)$ is the set of all continuous and bounded functions from $Y$ to $\mathbb{R}$.

3. Definition/Notation: Given $A \subset X$, then $\chi_{A}: X \rightarrow \mathbb{R}$ is called the characteristic function of $A$ if $\chi(a)=1$ for $a \in A$ and $\chi(a)=0$ for $a \notin A$. Characteristic functions are similarly defined on $B \subset Y$.

4. Definition: A map $S: X \rightarrow Y$ from a metric space $X$ to a metric space $Y$ is called a Borel map if the inverse image of every Borel (equivalently: open, equivalently: closed) set in $Y$ is a Borel set in $X$. 


\section{Proof:}

The fundamental observation and key to this proof is using characteristic functions on sets in $X, Y$, using characteristic functions to build up simple functions on sets in $X, Y$, and then using a sequence of simple functions converging pointwise to a continuous, bounded function on $Y$.

\section{Proof of $\Rightarrow$ :}

Lemma: Suppose $B \subset Y$ is measurable, $\chi_{B}$ is the characteristic function of $B$, and $S: X \rightarrow Y$ is a Borel map. Then the characteristic functions on $Y$ and $X$ are related by $\chi_{S^{-1}(B)}=\chi_{B} \circ S$.

Lemma Proof:

$$
\begin{gathered}
\chi_{S^{-1}(B)}(x)=1 \Longleftrightarrow x \in S^{-1}(B) \Longleftrightarrow S(x) \in B \\
\Longleftrightarrow \chi_{B}(S(x))=1 \Longleftrightarrow \chi_{B} \circ S(x)=1
\end{gathered}
$$

and

$$
\begin{gathered}
\chi_{S^{-1}(B)}(x)=0 \Longleftrightarrow x \notin S^{-1}(B) \Longleftrightarrow S(x) \notin B \\
\Longleftrightarrow \chi_{B}(S(x))=0 \Longleftrightarrow \chi_{B} \circ S(x)=0 .
\end{gathered}
$$

Thus, we have shown that $\chi_{S^{-1}(B)}=\chi_{B} \circ S$.

Suppose $B \subset Y$ is measurable and $\chi_{B}$ is the characteristic function of $B$. Then by definition of the Lebesgue integral:

$$
\nu(B)=\int_{Y} \chi_{B}(y) \nu(d y)
$$

Since $S: X \rightarrow Y$ is a Borel map and $B$ is measurable, $S^{-1}(B) \subset X$ is measureable. If $\chi_{S^{-1}(B)}$ is the characteristic function of $S^{-1}(B)$, then by definition of the Lebesgue integral and the property we just showed of the characteristic function:

$$
\mu\left(S^{-1}(B)\right)=\int_{X} \chi_{S^{-1}(B)}(x) \mu(d x)=\int_{X} \chi_{B}(S(x)) \mu(d x) .
$$

Since we assumed that $S_{\#} \mu=\nu$,

$$
\int_{X} \chi_{B}(S(x)) \mu(d x)=\mu\left(S^{-1}(B)\right)=\nu(B)=\int_{Y} \chi_{B}(y) \nu(d y) .
$$

The general strategy now will be the following steps. Step 1: Split the closure of the range of $\phi$ into $n$ disjoint pieces. Step 2: Approximate $p h i$ by a sum of $n+1$ characteristic functions on disjoint subsets of $X$ whose union is $X$. Step 3: Pass to the limit of the sequence $\left\{\phi_{n}\right\}$ as $n \rightarrow \infty$.

Step 1: Suppose $\phi \in C_{b}(Y)$. Then 


$$
-\infty<\inf _{y \in Y} \phi \leq \sup _{y \in Y} \phi<\infty
$$

So let $m=\inf _{y \in Y}$ and $M=\sup _{y \in Y}$ and define $L=M-m$. Then for $n \in \mathbb{N}$ and for $k \in \mathbb{N}$ such that $1 \leq k \leq n$, define:

$$
\begin{aligned}
Y_{n, k} & =\left\{y \in Y: m+\frac{(k-1) L}{n} \leq \phi(y)<m+\frac{k L}{n}\right\} \\
& =\quad \phi^{-1}\left(\left[m+\frac{(k-1) L}{n}, m+\frac{k L}{n}\right)\right)
\end{aligned}
$$

and define

$$
Y_{n, n+1}=\{y \in Y: \phi(y)=M\}=\phi^{-1}(\{M\}) .
$$

Now note that $\{M\}$ and $\left[m+\frac{(k-1) L}{n}, m+\frac{k L}{n}\right)$ are Borel sets in $\mathbb{R}$ and $\phi$ is a Borel map (since it is continuous) and so $Y_{n, k}$ are Borel sets for all $n$ and for all $1 \leq k \leq n+1$.

Further note that for all $n$ and for all $i \neq j$ such that $1 \leq i \leq n+1$ and $1 \leq j \leq n+1$ we have:

$$
Y_{n, i} \cap Y_{n, j}=\emptyset
$$

And finally note that for any $n$ :

$$
\cup_{k=1}^{n+1} Y_{n, k}=Y
$$

Step 2: Now define:

$$
C_{n, k}=m+\frac{(k-1) L}{n}
$$

And define:

$$
\begin{aligned}
& \phi_{n, k}: Y \rightarrow \mathbb{R} \text { by } \\
& \begin{aligned}
\phi_{n, k}(y) & =\left\{\begin{array}{cc}
C_{n, k} & \text { if } y \in Y_{n, k} \\
0 & \text { else }
\end{array}\right. \\
& =\quad C_{n, k} \chi_{Y_{n, k}} .
\end{aligned}
\end{aligned}
$$

Now from what was proven earlier (equation 1) about characteristic functions:

$$
\int_{X} \chi_{Y_{n, k}}(S(x)) \mu(d x)=\int_{Y} \chi_{Y_{n, k}}(y) \nu(d y)
$$


Thus:

$$
\int_{X} \phi_{n, k}(S(x)) \mu(d x)=\int_{Y} \phi_{n, k}(y) \nu(d y)
$$

Now let us define:

$$
\phi_{n}: Y \rightarrow \mathbb{R} \text { by } \phi_{n}=\sum_{k=1}^{n+1} \phi_{n, k} .
$$

And note that since the Lebesgue integral is linear and from what we just showed:

$$
\begin{aligned}
& \int_{X} \phi_{n}(S(x)) \mu(d x)=\int_{X} \sum_{k=1}^{n+1} \phi_{n, k}(S(x)) \mu(d x) \\
&=\sum_{k=1}^{n+1} \int_{X} \phi_{n, k}(S(x)) \mu(d x) \\
&=\sum_{k=1}^{n+1} \int_{Y} \phi_{n, k}(y) \nu(d y) \\
&=\int_{Y} \sum_{k=1}^{n+1} \phi_{n, k}(y) \nu(d y) \\
&= \\
& \int_{Y} \phi_{n}(y) \nu(d y) .
\end{aligned}
$$

Now suppose we have a given $n$ and select $x \in X$. Earlier we showed that for all $i \neq j$ such that $1 \leq i \leq n+1$ and $1 \leq j \leq n+1$ we have:

$$
Y_{n, i} \cap Y_{n, j}=\emptyset
$$

We also have that:

$$
\cup_{k=1}^{n+1} Y_{n, k}=Y \text {. }
$$

So $\exists ! k$ such that for the given $x \in X, S(x) \in Y_{n, k}$ and note that by the definition of $Y_{n, k}$ :

$$
\left|\phi_{n}(S(x))-\phi(S(x))\right|<\frac{1}{n}
$$

Note that for a given $n,\left|\phi_{n}(S(x))-\phi(S(x))\right|<\frac{1}{n}$ regardless of what $x \in X$ is chosen, thus:

$$
\begin{aligned}
\left|\int_{X}-\phi_{n}(S(x))+\phi(S(x)) \mu(d x)\right| & \leq & \int_{X}\left|-\phi_{n}(S(x))+\phi(S(x))\right| \mu(d x) \\
& \leq & \int_{X} \frac{1}{n} \mu(d x) \\
& = & \frac{1}{n} \mu(X) .
\end{aligned}
$$


Step 3: Now $\mu$ is a finite measure on $X$ by assumption, so

$$
\lim _{n \rightarrow \infty}\left|\int_{X}-\phi_{n}(S(x))+\phi(S(x)) \mu(d x)\right| \leq \lim _{n \rightarrow \infty} \frac{1}{n} \mu(X)=0 .
$$

Now, recall the following definition: a sequence of functions $\phi_{n}$ converges uniformly to a function $\phi$ if $\forall \epsilon>0, \exists N>0$ such that $\forall y \in Y,\left|\phi_{n}(y)-\phi(y)\right|<\epsilon \mid$.

So now note that in addition, the functions $\phi_{n}$ converge uniformly to $\phi$ and so

$$
\lim _{n \rightarrow \infty} \int_{Y} \phi_{n}(y) \nu(d y)=\int_{Y} \lim _{n \rightarrow \infty} \phi_{n}(y) \nu(d y)=\int_{Y} \phi(y) \nu(d y)
$$

So again by linearity of the integral and what we showed earlier:

$$
\begin{aligned}
\int_{X} \phi(S(x)) \mu(d x) & =\quad \int_{X}\left[\phi_{n}(S(x))-\phi_{n}(S(x))+\phi(S(x))\right] \mu(d x) \\
& =\int_{X} \phi_{n}(S(x)) \mu(d x)+\int_{X}\left[-\phi_{n}(S(x))+\phi(S(x))\right] \mu(d x) \\
& =\int_{Y} \phi_{n}(y) \nu(d y)+\int_{X}\left[-\phi_{n}(S(x))+\phi(S(x))\right] \mu(d x) .
\end{aligned}
$$

Using (2.1.3) and (2.1.4) and taking the limit as $n$ goes to $\infty$ of the right hand side,

$$
\begin{array}{ccc}
\int_{X} \phi(S(x)) \mu(d x) & =\lim _{n \rightarrow \infty}\left(\int_{Y} \phi_{n}(y) \nu(d y)+\int_{X}\left[-\phi_{n}(S(x))+\phi(S(x))\right] \mu(d x)\right) \\
& = & \lim _{n \rightarrow \infty} \int_{Y} \phi_{n}(y) \nu(d y)+ \\
& & \lim _{n \rightarrow \infty} \int_{X}\left[-\phi_{n}(S(x))+\phi(S(x))\right] \mu(d x) \\
= & \int_{Y} \phi(y) \nu(d y)+0
\end{array}
$$

Thus we have proven $\Rightarrow$ by showing what we intended to show, i.e. that for an arbitrary continuous bounded function $\phi$ from $Y$ to $\mathbb{R}$ we have $\int_{X} \phi(S(x)) \mu(d x)=\int_{Y} \phi(y) \nu(d y)$. 
Reminder of a topology result:

Definition: If $X$ is a topological space, then $X$ is normal if $\forall A, B \subset X$ such that $A, B$ are closed and $A \cap B=\emptyset$, then $\exists O_{A}, O_{B}$ open such that $A \subset O_{A}, B \subset O_{B}$ and $O_{A} \cap O_{B}=\emptyset$.

Urysohn's Lemma: If $X$ is a normal space (and all metric spaces are normal), $C \subset O \subset$ $X, C$ closed and $O$ open, then there exists a continuous function $f: X \rightarrow[0,1]$ such that $f(x)=1$ for all $x \in C$ and $f(x)=0$ for all $x \in X \backslash O$.

\section{Proof of $\Leftarrow$ :}

Suppose $B \subset Y$ is a closed set and given any $n$ integer with $n \geq 1$, define the open set

$$
O_{n}=\left\{y \in Y: \operatorname{dist}(y, B)<\frac{1}{n}\right\}
$$

Note that for all $n$ integer with $n \geq 1$, we have $B \subset O_{n}$. We also have that $O_{1} \supset O_{2} \supset$ $O_{3} \supset \ldots \supset O_{k} \supset \ldots$

And finally, we have that $\cap_{n=1}^{\infty} O_{n}=B$, which we show here:

(つ): Since $B \subset O_{n}$ for all $n$ integer with $n \geq 1$, we have that $B \subset \cap_{n=1}^{\infty} O_{n}$.

$(\subset)$ : Now suppose $y \in \cap_{n=1}^{\infty} O_{n}$. This implies that $y \in O_{n}$ for all $n$ integer with $n \geq 1$. By the definition of the sets $O_{n}$, this implies that $\operatorname{dist}(y, B)<\frac{1}{n}$ for all $n$ integer with $n \geq 1$. This implies that $\operatorname{dist}(y, B)=0$. But then by properties of closed sets in metric spaces, this implies that $y \in B$.

Thus we have shown $B \subset \cap_{n=1}^{\infty} O_{n}$ and $\cap_{n=1}^{\infty} O_{n} \subset B$, which together imply that $B=$ $\cap_{n=1}^{\infty} O_{n}$.

Now since $\nu$ is a finite measure (it is a probability measure), $\nu\left(O_{1}\right)<\infty$. Thus we can use the descending sequence of sets theorem for measures and conclude that $\lim _{n \rightarrow \infty} \nu\left(O_{n}\right)=$ $\nu\left(\cap_{n=1}^{\infty} O_{n}\right)=\nu(B)$.

So now for each $n$ integer with $n \geq 1$, apply Urysohn's Lemma to $O_{n}$ and $B$ to conclude there exists a continuous function $\phi_{n}: Y \rightarrow[0,1]$ such that $\phi_{n}(y)=1$ if $y \in B$ and $\phi_{n}(y)=0$ if $y \in Y \backslash O_{n}$.

We now claim that $\phi_{n} \rightarrow \chi_{B}$ pointwise as $n \rightarrow \infty$. Proof:

Suppose $y \in Y$. Then either $y \in B$ or $y \notin B$. If $y \in B$, then $\chi_{B}(y)=1$ and for all $n$ integer with $n \geq 1$, we have $\phi_{n}(y)=1$. Thus if $y \in B$, then for all $n$ integer with $n \geq 1$, we have $\phi_{n}(y)=\chi_{B}(y)$.

So now suppose $y \notin B$. Then since $B$ is closed, there exists $n_{0}$ integer with $n_{0} \geq 1$ such that $y \in Y \backslash O_{n}$ for all $n \geq n_{0}$. Thus for all $n \geq n_{0}$, we have $\phi_{n}(y)=0$. But also since $y \notin B$, we see that $\chi_{B}(y)=0$. So for all $n$ integer with $n \geq n_{0}$, we have $\phi_{n}(y)=\chi_{B}(y)$.

Thus we have shown that $\phi_{n} \rightarrow \chi_{B}$ pointwise as $n \rightarrow \infty$.

Now note that $\phi_{n} \in C_{b}(Y)$, so by assumption:

$$
\int_{X} \phi_{n}\left(S(x) \mu(d x)=\int_{Y} \phi_{n}(y) \nu(d y)\right.
$$


Now $Y=O_{n} \cup\left(Y \backslash O_{n}\right), O_{n} \cap\left(Y \backslash O_{n}\right)=\emptyset, O_{n}$ (being open) and $Y \backslash O_{n}$ (being closed) are both measurable, and $\phi_{n}(y)=0$ for all $y \in Y \backslash O_{n}$. Thus:

$$
\begin{aligned}
\int_{Y} \phi_{n}(y) \nu(d y) & = & \int_{O_{n}} \phi_{n}(y) \nu(d y)+\int_{Y \backslash O_{n}} \phi_{n}(y) \nu(d y) \\
& = & \int_{O_{n}} \phi_{n}(y) \nu(d y)+0 \\
& = & \int_{O_{n}} \phi_{n}(y) \nu(d y) .
\end{aligned}
$$

Next, since $\phi_{n}(y)=1$ for all $y \in B$ and since $O_{n}=B \cup\left(O_{n} \backslash B\right), B \cap\left(O_{n} \backslash B\right)=\emptyset$, and $B$ (closed) and $O_{n} \backslash B$ (open) are both measurable, we have:

$$
\begin{aligned}
\int_{O_{n}} \phi_{n}(y) \nu(d y) & =\int_{B} \phi_{n}(y) \nu(d y)+\int_{O_{n} \backslash B} \phi_{n}(y) \nu(d y) \\
& =\quad \int_{B} \nu(d y)+\int_{O_{n} \backslash B} \phi_{n}(y) \nu(d y) \\
& =\quad \nu(B)+\int_{O_{n} \backslash B} \phi_{n}(y) \nu(d y) .
\end{aligned}
$$

Now since $\phi_{n}$ is continuous and since $0 \leq \phi_{n}(y) \leq 1$ for all $y \in Y$, we see that:

$$
0 \leq \int_{O_{n} \backslash B} \phi_{n}(y) \nu(d y) \leq \int_{O_{n} \backslash B} \nu(d y)=\nu\left(O_{n} \backslash B\right) .
$$

Now since $O_{n}$ (open), $B$ (closed), $O_{n} \backslash B$ (open) are measurable and since $B \cup\left(O_{n} \backslash B\right)=O_{n}$ and $B \cap\left(O_{n} \backslash B\right)=\emptyset$, we have $\nu\left(O_{n}\right)=\nu\left(O_{n} \backslash B\right)+\nu(B)$. Combining this with the inequalities above, we have:

$$
0 \leq \int_{O_{n} \backslash B} \phi_{n}(y) \nu(d y) \leq \nu\left(O_{n}\right)-\nu(B)
$$

Now above, we showed that $\lim _{n \rightarrow \infty} \nu\left(O_{n}\right)=\nu(B)$ and so as $n \rightarrow \infty$, we have that $\int_{O_{n} \backslash B} \phi_{n}(y) \nu(d y) \rightarrow 0$.

Combining this with (2.1.5), (2.1.6), and (2.1.7), we have that as $n \rightarrow \infty$, we have:

$$
\int_{X} \phi_{n}(S(x)) \mu(d x)=\int_{Y} \phi(y) \nu(d y) \rightarrow \nu(B) .
$$

Next $S^{-1}(B), S^{-1}\left(O_{n}\right) \backslash S^{-1}(B), X \backslash S^{-1}\left(O_{n}\right)$ are measurable, disjoint, and have union equal to $X$. In addition, for $x \in S^{-1}(B)$ we have $\phi_{n}(S(x))=1$ and for $x \in X \backslash S^{-1}\left(O_{n}\right)$ we have $\phi_{n}(S(x))=0$. Thus: 


$$
\begin{array}{ccc}
\int_{X} \phi_{n}(S(x)) \mu(d x)= & \int_{S^{-1}(B)} \phi_{n}(S(x)) \mu(d x)+ \\
& \int_{S^{-1}\left(O_{n}\right) \backslash S^{-1}(B)} \phi_{n}(S(x)) \mu(d x)+ \\
& & \int_{X \backslash S^{-1}\left(O_{n}\right)} \phi_{n}(S(x)) \mu(d x) \\
& \int_{S^{-1}(B)} \mu(d x)+ \\
= & \int_{X \backslash S^{-1}\left(O_{n}\right) \backslash S^{-1}(B)} \phi_{n}(S(x)) \mu(d x)+ \\
& \left.\int_{S^{-1}\left(O_{n}\right) \backslash S^{-1}(B)} \phi_{n}(B)\right)+
\end{array}
$$

Again, since $\phi_{n}$ is continuous and for all $y \in Y$ we have $0 \leq \phi_{n}(y) \leq 1$, this results in:

$$
\begin{aligned}
0 & \leq & \int_{S^{-1}\left(O_{n}\right) \backslash S^{-1}(B)} \phi_{n}(S(x)) \mu(d x) \\
& \leq & \int_{S^{-1}\left(O_{n}\right) \backslash S^{-1}(B)} \mu(d x) \\
& = & \mu\left(S^{-1}\left(O_{n}\right) \backslash S^{-1}(B)\right) .
\end{aligned}
$$

Now note that $\mu\left(S^{-1}\left(O_{1}\right) \backslash S^{-1}(B)\right)<\infty$ and $\mu\left(S^{-1}\left(O_{1}\right) \backslash S^{-1}(B)\right) \supset \mu\left(S^{-1}\left(O_{2}\right) \backslash\right.$ $\left.S^{-1}(B)\right) \supset \ldots \supset \mu\left(S^{-1}\left(O_{k}\right) \backslash S^{-1}(B)\right) \supset \ldots$

Now $\cap_{n=1}^{\infty}\left(S^{-1}\left(O_{n}\right) \backslash S^{-1}(B)\right)=\emptyset$ and properties of measures imply that as $k \rightarrow \infty$ :

$$
\mu\left(S^{-1}\left(O_{k}\right) \backslash S^{-1}(B)\right) \rightarrow \mu\left(\cap_{n=1}^{\infty}\left(S^{-1}\left(O_{n}\right) \backslash S^{-1}(B)\right)\right)=\mu(\emptyset)=0 .
$$

Combining results (2.1.8), (2.1.9), (2.1.10), and (2.1.11) from above, we have that as $n \rightarrow \infty, \int_{X} \phi_{n}(S(x)) \mu(d x) \rightarrow \nu(B)$ and $\int_{X} \phi_{n}\left(S(x) \mu(d x) \rightarrow \mu\left(S^{-1}(B)\right)\right.$, and so as $n \rightarrow \infty$ we have $\nu(B)=\mu\left(S^{-1}(B)\right)$.

Thus we have shown that if $B$ is a closed subset of $Y$ and if $\forall \phi \in C_{b}(Y), \int_{X} \phi(S(x)) \mu(d x)=$ $\int_{Y} \phi(y) \nu(d y)$, we can conclude that $\nu(B)=\mu\left(S^{-1}(B)\right)$.

What remains to show is the result for a general measurable set $B$.

Now suppose $B \subset Y$ and define $p(B)=\mu\left(S^{-1}(B)\right.$. Then since $\mu$ is a Borel measure on $X$ and $S: X \rightarrow Y$ is a Borel map, $p$ is a Borel measure on $X$. From what we just showed above, we also know for all $B$ closed subsets of $Y$ we have $p(B)=\nu(B)$.

Now suppose $O$ is an open subset of $Y$. Then by the definition of a closed set in a topological space, $C=Y \backslash O$ is a closed subset of $Y$. But then by the result above, we have:

$$
p(Y \backslash O)=\nu(Y \backslash O)
$$

Now since $p$ and $\nu$ are Borel measures on $Y$, we have that $p(Y \backslash O)=p(Y)-p(O)$ and $\nu(Y \backslash O)=\nu(Y)-\nu(O)$ and so we see that: 


$$
p(Y)-p(O)=\nu(Y)-\nu(O)
$$

Now since $Y$ is the entire space, it is closed and so as we showed, $p(Y)=\nu(Y)$.

We must be a little careful here, since we can only conclude that $p(O)=\nu(O)$ if $p(Y)=$ $\nu(Y)$ is finite. But we assumed at the beginning that $\nu$ is a finite measure on $Y$ and so this is true. Thus we conclude that $p(O)=\nu(O)$ for any open subset of $Y$.

Now any Borel subset $B$ of $Y$ is in the sigma algebra generated by the open subsets of $Y$ and thus $\nu(B)=p(B)=\mu\left(S^{-1}(B)\right)$.

Therefore $S_{\#} \mu=\nu$ and we are done.

\subsection{Optimal Transport}

Next we recall several definitions and results from Optimal Transport. Again, these can be found in any standard reference such as [13].

Definition 2.2.1. Suppose $\pi \in \mathcal{P}_{2}(\mathbb{R} \times \mathbb{R}) . \pi$ is said to have first marginal $\mu \in \mathcal{P}_{2}(\mathbb{R})$ if $\pi(A \times \mathbb{R})=\mu(A)$ for all measurable sets $A$ of $\mathbb{R}$. $\pi$ is said to have second marginal $\nu \in \mathcal{P}_{2}(\mathbb{R})$ if $\pi(\mathbb{R} \times B)=\nu(B)$ for all measurable sets $B$ of $\mathbb{R}$.

Let $X$ and $Y$ be two separable metric spaces such that any probability measure on $\mathrm{X}$ (or $\mathrm{Y}$ ) is a Radon measure (i.e. they are Radon spaces). Let $c: X \times Y \rightarrow[0, \infty]$ be a Borelmeasurable function (often called the cost function). Given probability measures $\mu$ on $X$ and $n u$ on $Y$, Monge's formulation of the optimal transportation problem is to find a transport map $T: X \rightarrow Y$ that realizes the infimum

$$
\inf \left\{\int_{X} c(x, T(x)) d \mu(x): T_{\# \mu}=\nu\right\}
$$

Definition 2.2.2. A map that attains this infimum is called an Optimal Transport Map.

This formulation allows the possibility that there is no such $T$ and so Kantorovich developed the more general formulation which follows:

Given measures $\mu$ on $X, \nu$ on $Y$, and $\pi$ on $X \times Y, \pi$ is said to have (first) marginal $\mu$ if $\pi(A \times Y)=\mu(A)$ for all measurable subsets $A$ of $X . \pi$ is said to have (second) marginal $\nu$ if $\pi(X \times B)=\nu(B)$ for all measurable subsets $B$ of $Y$.

The Kantorovich formulation of the optimal transport problem is the following. Let $c$ : $X \times Y \rightarrow[0, \infty]$ be a Borel-measurable function (often called the cost function). Let $\Pi(\mu, \nu)$ be the set of all measures on $X \times Y$ such that if $\pi \in \Pi$ then $\pi$ has first marginal $\mu$ and second marginal $\nu$. Find the $\pi \in \Pi$ that minimizes:

$$
I(\pi)=\int_{X \times Y} c(x, y) d \pi(x, y) .
$$


Theorem 2.2.1. Suppose $c$ is a lower semi-continuous cost function. Then the Kantorovich formulation of the optimal transportation problem admits a minimizer.

A useful duality problem exists for the Kantorovich formulation and is the following:

Theorem 2.2.2. Let $c: X \times Y \rightarrow[0, \infty]$ be a Borel-measurable function (often called the cost function). Let $\Pi(\mu, \nu)$ be the set of all measures on $X \times Y$ such that if $\pi \in \Pi$ then $\pi$ has first marginal $\mu$ and second marginal $\nu$. Then given all bounded and continuous $\phi: X \rightarrow \mathbb{R}, \psi$ : $Y \rightarrow \mathbb{R}$ such that $\phi(x)+\psi(y) \leq c(x, y)$, the minimizer of the Kantorovich formulation can be found by:

$$
I(\pi)=\sup \left\{\int_{X} \phi(x) d \mu(x)+\int_{Y} \psi(y) d \nu(y)\right\}
$$

where the supremum is taken over $\phi, \psi$.

Definition 2.2.3. Given $\mu, \nu \in \mathcal{P}_{2}(\mathbb{R})$, the Wasserstein distance, $W_{2}^{2}(\mu, \nu)$, between $\mu$ and $\nu$ is defined as:

$$
W_{2}^{2}(\mu, \nu)=\inf \left\{\int_{\mathbb{R} \times \mathbb{R}}|x-y|^{2} \pi(d x, d y): \pi \in \mathcal{P}(\mathbb{R} \times \mathbb{R}) \text { and } \pi \text { has marginals } \mu, \nu\right\} .
$$

Definition 2.2.4. Given $\mu \in \mathcal{P}(\mathbb{R})$ and a Borel map $X: \mathbb{R} \rightarrow \mathbb{R}$, the push forward $\nu$ of $\mu$ by $X$, denoted $\nu=X_{\#} \mu$ is a measure $\nu \in \mathcal{P}(\mathbb{R})$ defined as $\nu(B)=\mu\left(X^{-1}(B)\right)$ for all Borel subsets $B$ of $\mathbb{R}$.

Definition 2.2.5. Suppose $\pi \in \mathcal{P}_{2}(\mathbb{R} \times \mathbb{R}) . \pi$ is said to have first marginal $\mu \in \mathcal{P}_{2}(\mathbb{R})$ if $\pi(A \times \mathbb{R})=\mu(A)$ for all measurable sets $A$ of $\mathbb{R}$. $\pi$ is said to have second marginal $\nu \in \mathcal{P}_{2}(\mathbb{R})$ if $\pi(\mathbb{R} \times B)=\nu(B)$ for all measurable sets $B$ of $\mathbb{R}$.

Definition 2.2.6. Given $\mu, \nu \in \mathcal{P}_{2}(\mathbb{R})$, the Wasserstein distance, $W_{2}^{2}(\mu, \nu)$, between $\mu$ and $\nu$ is defined as:

$$
W_{2}^{2}(\mu, \nu)=\inf \left\{\int_{\mathbb{R} \times \mathbb{R}}|x-y|^{2} \pi(d x, d y): \pi \in \mathcal{P}(\mathbb{R} \times \mathbb{R}) \text { and } \pi \text { has marginals } \mu, \nu\right\} .
$$




\section{Chapter 3}

\section{Lagrangian Description of Pressureless Euler System Solutions}

\subsection{SPF solutions to (PE-IC)}

The goal of this section is to prove that SPF solutions to (PE-IC) satisfy (SICE).

Proposition 3.1.1. Suppose

1. $\rho_{0} \in \mathcal{P}_{2}(\mathbb{R})$,

2. $v_{0} \in L^{2}\left(\rho_{0}\right)$, i.e. $v_{0}$ is measurable and $\int_{\mathbb{R}} v_{0}^{2} d \rho_{0}=: C^{2}<\infty$,

3. $X:[0, \infty) \rightarrow L^{2}\left(\rho_{0}\right)$ is continuous at $t=0, X(0, \cdot)=\operatorname{Id} \rho_{0}$ a.e., and $\rho_{t}:=X(t, \cdot)_{\#} \rho_{0}$,

4. For each $t>0, v(t, \cdot)=v_{t}$ is the unique element of $L^{2}\left(\rho_{t}\right)$ for which $v(t, X(t, \cdot))=$ $\mathbb{E}_{\rho_{0}}\left[v_{0} \mid X(t, \cdot)\right]$.

Then

$$
\int_{\mathbb{R}} v(t, y) \phi(y) \rho(t, d y) \underset{t \rightarrow 0^{+}}{\longrightarrow} \int_{\mathbb{R}} v_{0}(y) \phi(y) \rho_{0}(d y) \text { for all } \phi \in C_{b}(\mathbb{R}) .
$$

and (SICE) holds.

Proof. Note that

$$
\begin{aligned}
\int_{\mathbb{R}} v_{t}(y) \phi(y) \rho_{t}(d y)-\int_{\mathbb{R}} v_{0}(y) \phi(y) \rho_{0}(d y) & =\int_{\mathbb{R}} v_{t}\left(X_{t}(y)\right) \phi\left(X_{t}(y)\right) \rho_{0}(d y)-\int_{\mathbb{R}} v_{0}(y) \phi(y) \rho_{0}(d y) \\
& =\int_{\mathbb{R}} v_{0}(y) \phi\left(X_{t}(y)\right) \rho_{0}(d y)-\int_{\mathbb{R}} v_{0}(y) \phi(y) \rho_{0}(d y) \\
& =\int_{\mathbb{R}} v_{0}(y)\left[\phi\left(X_{t}(y)\right)-\phi(y)\right] \rho_{0}(d y) .
\end{aligned}
$$

Now 


$$
\begin{aligned}
\left|\int_{\mathbb{R}} v_{0}(y)[\phi(X(t, y))-\phi(y)] \rho_{0}(d y)\right| & \leq \int_{\mathbb{R}}\left|v_{0}(y)[\phi(X(t, y))-\phi(y)]\right| \rho_{0}(d y) \\
& \leq\left(\int_{\mathbb{R}} v_{0}^{2} d \rho_{0}\right)^{1 / 2}\left(\int_{\mathbb{R}}[\phi(X(t, y))-\phi(y)]^{2} \rho_{0}(d y)\right)^{1 / 2} \\
& =C\left(\int_{\mathbb{R}}[\phi(X(t, y))-\phi(y)]^{2} \rho_{0}(d y)\right)^{1 / 2} .
\end{aligned}
$$

But $\phi$ is bounded, so $|\phi(X(t, y))-\phi(y)|$ is bounded, and so $[\phi(X(t, y))-\phi(y)]^{2}$ is bounded. In addition, as $t \rightarrow 0^{+}, \phi(X(t, y)) \rightarrow \phi(y)$ for $\rho_{0}$ a.e. $y$. Thus, by the Dominated Convergence Theorem, $\int_{\mathbb{R}}[\phi(X(t, y))-\phi(y)]^{2} \rho_{0}(d y) \rightarrow 0$ as $t \rightarrow 0^{+}$, which finishes the proof of the first statement.

Let us now prove the second. Consider an arbitrary sequence $\left\{t_{n}\right\}_{n \geq 1}$ such that $t_{n} \rightarrow 0^{+}$. Define $v_{n}:=v\left(t_{n}, \cdot\right), X_{n}:=X\left(t_{n}, \cdot\right), \rho_{n}:=X_{n \#} \rho_{0}$. Note that the hypothesis on $X$ implies $X\left(t_{n}, y\right) \rightarrow y$ for $\rho_{0}$ a.e. $y$. So what we need to show is that

$$
\int_{\mathbb{R}} v_{n}^{2}\left(X_{n}(y)\right) \phi\left(X_{n}(y)\right) \rho_{0}(d y) \underset{n \rightarrow \infty}{\longrightarrow} \int_{\mathbb{R}} v_{0}^{2}(y) \phi(y) \rho_{0}(d y) \quad \forall \phi \in C_{b}(\mathbb{R}) .
$$

\section{Step 1:}

By the definition of $v_{n}$, which gives $v_{n} \circ X_{n}$ as the conditional expectation of $v_{0}$ with respect to $\rho_{0}$ given $X_{n}$, we have

$$
\int_{\mathbb{R}} v_{n}\left(X_{n}(y)\right) \zeta\left(X_{n}(y)\right) \rho_{0}(d y)=\int_{\mathbb{R}} v_{0}(y) \zeta\left(X_{n}(y)\right) \rho_{0}(d y)
$$

for any $\zeta \in L^{2}\left(\rho_{n}\right)$. First, the identity above implies $\left\|v_{n}\right\|_{L^{2}\left(\rho_{n}\right)} \leq\left\|v_{0}\right\|_{L^{2}\left(\rho_{0}\right)}$ for all $n \geq 1$. Secondly, by letting $\zeta=\phi v_{n}$, for some $\phi \in C_{b}(\mathbb{R})$, we have $\zeta \in L^{2}\left(\rho_{n}\right)$ and so

$$
\begin{aligned}
\int_{\mathbb{R}}\left(v_{n} \circ X_{n}\right)^{2}\left(\phi \circ X_{n}\right) d \rho_{0} & =\int_{\mathbb{R}}\left(v_{n} \circ X_{n}\right)\left(v_{n} \circ X_{n}\right)\left(\phi \circ X_{n}\right) d \rho_{0} \\
& =\int_{\mathbb{R}} v_{0}\left(v_{n} \circ X_{n}\right)\left(\phi \circ X_{n}\right) d \rho_{0}
\end{aligned}
$$

for all $\phi \in C_{b}(\mathbb{R})$. Thus, showing (3.1.2) is equivalent to showing

$$
\int_{\mathbb{R}} v_{0}\left(v_{n} \circ X_{n}\right)\left(\phi \circ X_{n}\right) d \rho_{0} \underset{n \rightarrow \infty}{\longrightarrow} \int_{\mathbb{R}} v_{0}^{2} \phi d \rho_{0} \quad \forall \phi \in C_{b}(\mathbb{R}) .
$$

\section{Step 2:}

We have $v_{0} \in L^{2}\left(\rho_{0}\right)$, so $v_{0}^{2}<\infty \rho_{0}$ a.e. Also $X_{n} \rightarrow \operatorname{Id} \rho_{0}$ a.e. and so $\phi \circ X_{n} \underset{n \rightarrow \infty}{\longrightarrow} \phi \rho_{0}$ a.e. Thus, $v_{0}^{2}\left[\phi \circ X_{n}-\phi\right]^{2} \underset{n \rightarrow \infty}{\longrightarrow} 0 \rho_{0}$ a.e. Since $\phi \in C_{b}(\mathbb{R})$ and $v_{0} \in L^{2}\left(\rho_{0}\right)$, by Dominated Convergence we infer

$$
\left\|v_{0}\left[\phi \circ X_{n}-\phi\right]\right\|_{L^{2}\left(\rho_{0}\right)} \underset{n \rightarrow \infty}{\longrightarrow} 0 .
$$


Noting that

$$
\begin{aligned}
\left|\int_{\mathbb{R}} v_{0}\left(v_{n} \circ X_{n}\right)\left[\phi \circ X_{n}-\phi\right] d \rho_{0}\right| & \leq\left\|v_{n} \circ X_{n}\right\|_{L^{2}\left(\rho_{0}\right)}\left\|v_{0}\left[\phi \circ X_{n}-\phi\right]\right\|_{L^{2}\left(\rho_{0}\right)} \\
& \leq\left\|v_{0}\right\|_{L^{2}\left(\rho_{0}\right)}\left\|v_{0}\left[\phi \circ X_{n}-\phi\right]\right\|_{L^{2}\left(\rho_{0}\right)}
\end{aligned}
$$

we get from (3.1.5) that

$$
\int_{\mathbb{R}} v_{0}\left(v_{n} \circ X_{n}\right)\left(\phi \circ X_{n}\right) d \rho_{0}-\int_{\mathbb{R}} v_{0}\left(v_{n} \circ X_{n}\right) \phi d \rho_{0} \underset{n \rightarrow \infty}{\longrightarrow} 0 .
$$

Thus, to show (3.1.4), it suffices to show

$$
\int_{\mathbb{R}} v_{0}\left(v_{n} \circ X_{n}\right) \phi d \rho_{0} \underset{n \rightarrow \infty}{\longrightarrow} \int_{\mathbb{R}} v_{0}^{2} \phi d \rho_{0} \quad \forall \phi \in C_{c}^{\infty}(\mathbb{R}) .
$$

\section{Step 3:}

Note that (3.1.1) (already proved above) translates to

$$
\int_{\mathbb{R}} v_{n}\left(X_{n}(y)\right) \phi\left(X_{n}(y)\right) \rho_{0}(d y) \underset{n \rightarrow \infty}{\longrightarrow} \int_{\mathbb{R}} v_{0}(y) \phi(y) \rho_{0}(d y) \quad \forall \phi \in C_{b}(\mathbb{R}) .
$$

Thus, given $\phi$ and $\epsilon$, we can choose $N_{1}$ such that $\forall n>N_{1}$,

$$
\left|\int_{\mathbb{R}} v_{n}\left(X_{n}(y)\right) \phi\left(X_{n}(y)\right) \rho_{0}(d y)-\int_{\mathbb{R}} v_{0}(y) \phi(y) \rho_{0}(d y)\right|<\frac{\epsilon}{4} .
$$

Now:

$$
\begin{aligned}
\mid \int_{\mathbb{R}} v_{n}\left(X_{n}(y)\right) \phi\left(X_{n}(y)\right) \rho_{0}(d y) & \\
-\int_{\mathbb{R}} v_{n}\left(X_{n}(y)\right) \phi(y) \rho_{0}(d y) \mid & =\mid \int_{\mathbb{R}} v_{n}\left(X_{n}(y)\right)\left[\phi\left(X_{n}(y)\right)-\phi(y) \rho_{0}(d y) \mid\right. \\
& \leq\left\|v_{n} \circ X_{n}\right\|_{L^{2}\left(\rho_{0}\right)}\left\|\phi \circ X_{n}-\phi\right\|_{L^{2}\left(\rho_{0}\right)} \\
& =\left\|v_{n}\right\|_{L^{2}\left(\rho_{n}\right)}\left\|\phi \circ X_{n}-\phi\right\|_{L^{2}\left(\rho_{0}\right)} \\
& \leq\left\|v_{0}\right\|_{L^{2}\left(\rho_{0}\right)}\left\|\phi \circ X_{n}-\phi\right\|_{L^{2}\left(\rho_{0}\right)} .
\end{aligned}
$$

But we have seen that

$$
\left\|\phi \circ X_{n}-\phi\right\|_{L^{2}\left(\rho_{0}\right)} \underset{n \rightarrow \infty}{\longrightarrow} 0,
$$

so, given $\phi$ and $\epsilon>0$, we can choose $N_{2}$ such that $\forall n>N_{2}$,

$$
\left|\int_{\mathbb{R}} v_{n}\left(X_{n}(y)\right) \phi\left(X_{n}(y)\right) \rho_{0}(d y)-\int_{\mathbb{R}} v_{n}\left(X_{n}(y)\right) \phi(y) \rho_{0}(d y)\right|<\frac{\epsilon}{4} .
$$


So given $\epsilon>0$ and $\phi$, we can choose $N$ to be the maximum of $N_{1}$ and $N_{2}$. Then for all $n>N$, the triangle inequality yields

$$
\left|\int_{\mathbb{R}} v_{0}(y) \phi\left(X_{n}(y)\right) \rho_{0}(d y)-\int_{\mathbb{R}} v_{n}\left(X_{n}(y)\right) \phi(y) \rho_{0}(d y)\right|<\frac{\epsilon}{2} .
$$

Next, suppose $\zeta \in L^{2}\left(\rho_{0}\right)$. If $v_{0}=0, \rho_{0}$-a.e., then assumption (4) implies $v_{n}=0, \rho_{n}$-a.e. so there is nothing to prove; so we can assume $\left\|v_{0}\right\|_{L^{2}\left(\rho_{0}\right)}>0$. Then, given $\epsilon>0$, there exists $\zeta_{\epsilon} \in C_{c}^{\infty}(\mathbb{R})$ such that

$$
\left\|\zeta_{\epsilon}-\zeta\right\|_{L^{2}\left(\rho_{0}\right)}<\frac{\epsilon}{4\left\|v_{0}\right\|_{L^{2}\left(\rho_{0}\right)}}
$$

which yields

$$
\begin{aligned}
\left|\int_{\mathbb{R}} v_{n}\left(X_{n}(y)\right)\left[\zeta(y)-\zeta_{\epsilon}(y)\right] \rho_{0}(d y)\right| & \leq\left\|v_{n} \circ X_{n}\right\|_{L^{2}\left(\rho_{0}\right)}\left\|\zeta_{\epsilon}-\zeta\right\|_{L^{2}\left(\rho_{0}\right)} \\
& =\left\|v_{n}\right\|_{L^{2}\left(\rho_{n}\right)}\left\|\zeta_{\epsilon}-\zeta\right\|_{L^{2}\left(\rho_{0}\right)} \\
& \leq\left\|v_{0}\right\|_{L^{2}\left(\rho_{0}\right)}\left\|\zeta_{\epsilon}-\zeta\right\|_{L^{2}\left(\rho_{0}\right)}<\frac{\epsilon}{4} .
\end{aligned}
$$

Also:

$$
\left|\int_{\mathbb{R}} v_{0}(y)\left[\zeta(y)-\zeta_{\epsilon}(y)\right] \rho_{0}(d y)\right| \leq\left\|v_{0}\right\|_{L^{2}\left(\rho_{0}\right)}\left\|\zeta_{\epsilon}-\zeta\right\|_{L^{2}\left(\rho_{0}\right)}<\frac{\epsilon}{4}
$$

Note that these last two estimates are independent of $n$. Choosing $\epsilon>0$, we can see that we can choose $\zeta_{\epsilon}$ such that (3.1.8) and (3.1.9) are satisfied. Then given $\epsilon$ and $\zeta_{\epsilon}$, we can see using (3.1.7) that we can choose $N$ such that for all $n>N$

$$
\left|\int_{\mathbb{R}} v_{n}\left(X_{n}(y)\right) \zeta(y) \rho_{0}(d y)-\int_{\mathbb{R}} v_{0}(y) \zeta(y) \rho_{0}(d y)\right|<\frac{\epsilon}{4}+\frac{\epsilon}{2}+\frac{\epsilon}{4}=\epsilon .
$$

Thus, for all $\zeta \in L^{2}\left(\rho_{0}\right)$

$$
\int_{\mathbb{R}} v_{n}\left(X_{n}(y)\right) \zeta(y) \rho_{0}(d y)-\int_{\mathbb{R}} v_{0}(y) \zeta(y) \rho_{0}(d y) \underset{n \rightarrow \infty}{\longrightarrow} 0 .
$$

Choosing $\zeta=v_{0} \phi$ and using the result above, we get

$$
\int_{\mathbb{R}} v_{0}(y) v_{n}\left(X_{n}(y)\right) \phi(y) \rho_{0}(d y) \underset{n \rightarrow \infty}{\longrightarrow} \int_{\mathbb{R}} v_{0}^{2}(y) \phi(y) \rho_{0}(d y) \quad \forall \phi \in C_{b}(\mathbb{R}) .
$$

Thus, we have shown (3.1.6), so we are done.

As for existence of SPF solutions, we will use (and, ultimately, extend) some results from Hynd [7]. We collect them in the statement below; all except part (3), which is a direct consequence of Proposition 3.1.1.

Theorem 3.1.1. Suppose $\rho_{0} \in \mathcal{P}_{2}(\mathbb{R})$ and $v_{0}: \mathbb{R} \rightarrow \mathbb{R}$ is absolutely continuous. Then there exists a solution $X$ to (SPF-IC). (Note that this solution $X$ is interpreted as a Lagrangian flow for the Sticky Particles System.) If $\rho(t, \cdot):=X(t, \cdot)_{\#} \rho_{0}$, then $(\rho, v)$ solves $(P E-I C)$ and satisfies: 
1. For Lebesgue almost every $t, s \geq 0$ with $s \leq t$, the non-increasing energy condition:

$$
\int_{\mathbb{R}} v^{2}(t, y) \rho(t, d y) \leq \int_{\mathbb{R}} v^{2}(s, y) \rho(s, d y) \leq \int_{\mathbb{R}} v_{0}^{2}(y) \rho_{0}(d y) .
$$

2. The pair $(\rho, v)$ satisfies (Oleinik).

3. The pair $(\rho, v)$ satisfies (SICE).

Ultimately, we will show that if $v_{0}$ is also right-continuous and bounded, then this SPF solution is unique and coincides with the SCL solution.

\subsection{SCL solutions to (PE-IC)}

The results from [10] we wish to call on are gathered here:

Theorem 3.2.1. Let $\rho_{0} \in \mathcal{P}_{2}(\mathbb{R})$ and let $M_{0}$ be the right-continuous cumulative distribution function of $\rho_{0}$. Let $v_{0} \in L^{2}\left(\rho_{0}\right)$. Then:

(i) There exists a Borel function $M:[0, \infty) \times \mathbb{R} \rightarrow[0,1]$ such that $M$ is the (unique) entropy (in the sense of Kruzhkov) solution to (SCL-IC).

(ii) For each $t \geq 0, M(t, \cdot)$ is non-decreasing and the right-continuous cumulative distribution function of a Borel probability $\rho(t, \cdot)$, and the distributional spatial derivative $\partial_{y} M(t, \cdot)=$ $\rho(t, \cdot), \partial_{y}[F(M(t, \cdot))]=v(t, \cdot) \rho(t, \cdot)$.

(iii) The pair $(\rho, v)$ is a solution to (PE-IC), called the SCL solution.

(iv) If $\left(\rho_{1}, v_{1}\right)$ and $\left(\rho_{2}, v_{2}\right)$ are the $S C L$ solutions to (PE-IC) for initial $\rho_{1}^{0}, \rho_{2}^{0} \in \mathcal{P}_{2}(\mathbb{R})$ and $v_{1}^{0} \in L^{2}\left(\rho_{1}^{0}\right), v_{2}^{0} \in L^{2}\left(\rho_{2}^{0}\right)$, then for all $t \geq 0$ one has

$$
W_{2}\left(\rho_{1}(t, \cdot), \rho_{2}(t, \cdot)\right) \leq W_{2}\left(\rho_{1}^{0}, \rho_{2}^{0}\right)+t\left\|v_{1}^{0} \circ N_{1}^{0}-v_{2}^{0} \circ N_{2}^{0}\right\|_{L^{2}(0,1)},
$$

where $N_{i}^{0}$ is the optimal map pushing $\left.\mathcal{L}^{1}\right|_{(0,1)}$ forward to $\rho_{i}^{0}, i=1,2$.

Note that (3.2.1) implies the following stability result:

Corollary 3.2.1. Suppose $\rho_{0} \in \mathcal{P}_{2}(\mathbb{R})$ and $v_{0}, \tilde{v}_{0} \in L^{2}\left(\rho_{0}\right)$ and let $(\rho, v),(\tilde{\rho}, \tilde{v})$ be the $S C L$ solutions to $(P E-I C)$ for the initial data $\left(\rho_{0}, v_{0}\right)$ and $\left(\rho_{0}, \tilde{v}_{0}\right)$, respectively. Then

$$
W_{2}(\rho(t, \cdot), \tilde{\rho}(t, \cdot)) \leq t\left\|v_{0}-\tilde{v}_{0}\right\|_{L^{2}\left(\rho_{0}\right)} \text { for all } t \geq 0 .
$$

Additionally, [10] proves the following:

Proposition 3.2.1. Suppose $\rho_{0} \in \mathcal{P}_{2}(\mathbb{R})$ and $v_{0} \in L^{2}\left(\rho_{0}\right)$. The $S C L$ solution $(\rho, v)$ to (PE-IC) satisfies:

1. the (Oleinik) condition;

2. the (SICE) condition; 
3. for Lebesgue almost every $t, s \geq 0$ with $s \leq t$, the non-increasing energy condition:

$$
\|v(t, \cdot)\|_{L^{2}(\rho(t, \cdot))} \leq\|v(s, \cdot)\|_{L^{2}(\rho(s, \cdot))}
$$

4. there exists a real constant $L$ for which

$$
W_{2}(\rho(s, \cdot), \rho(t, \cdot)) \leq L|t-s| \text { for all } s, t \geq 0
$$

We now prove a result which will be useful later.

Lemma 3.2.1. Suppose $\rho_{0} \in \mathcal{P}_{2}(\mathbb{R})$ and $v_{0} \in L^{2}\left(\rho_{0}\right)$. Let $\left\{v_{0, n}\right\}_{n \geq 1} \in L^{2}\left(\rho_{0}\right)$ converge to $v_{0}$ in $L^{2}\left(\rho_{0}\right)$, and let $\left(\rho_{n}, v_{n}\right)$ be the $S C L$ solution to (PE-IC) with initial data $\left(\rho_{0}, v_{0, n}\right)$. Let $(\rho, v)$ the $S C L$ solution corresponding to $\left(\rho_{0}, v_{0}\right)$. Then

$$
\int_{\mathbb{R}} v_{n}(t, y) \phi(y) \rho_{n}(t, d y) \underset{n \rightarrow \infty}{\longrightarrow} \int_{\mathbb{R}} v(t, y) \phi(y) \rho(t, d y) \text { for all } t \geq 0, \phi \in C_{b}(\mathbb{R}) .
$$

Proof. From (3.2.2) we get that $\rho_{n}(t)$ converges to $\rho(t)$ in the Wasserstein distance. Let $\phi \in C_{c}^{1}(\mathbb{R})$. We have

$$
\int_{\mathbb{R}} v_{n}(t, y) \phi(y) \rho_{n}(t, d y)=-\int_{\mathbb{R}} \phi^{\prime}(y) F_{n}\left(M_{n}(t, y)\right) d y
$$

and

$$
\int_{\mathbb{R}} v(t, y) \phi(y) \rho(t, d y)=-\int_{\mathbb{R}} \phi^{\prime}(y) F(M(t, y)) d y .
$$

Since $F_{n}$ converges uniformly to $F$ on $[0,1]$, we only need to analyze the convergence of $F\left(M_{n}(t, \cdot)\right)$ to $F(M(t, \cdot))$. From

$$
\left\|M_{n}(t, \cdot)-M(t, \cdot)\right\|_{L^{1}(\mathbb{R})}=W_{1}\left(\rho_{n}(t, \cdot), \rho(t, \cdot)\right) \leq W_{2}\left(\rho_{n}(t, \cdot), \rho(t, \cdot)\right),
$$

we deduce that, up to subsequence, $M_{n}(t, \cdot)$ converges to $M(t, \cdot)$ Lebesgue a.e. on $\mathbb{R}$. But $F$ is continuous and bounded, so, up to that sequence,

$$
\int_{\mathbb{R}}\left|\phi^{\prime}(y)\right|\left|F\left(M_{n}(t, y)\right)-F(M(t, y))\right| d y \underset{n \rightarrow \infty}{\longrightarrow} 0
$$

and so (3.2.3) is proved for $\phi \in C_{c}^{1}(\mathbb{R})$ (and for the whole sequence, since the limit is independent of the subsequence extracted above). Now let $\phi \in C_{b}(\mathbb{R})$ and $\left\{\phi_{m}\right\}_{m \geq 1} \subset C_{c}^{1}(\mathbb{R})$ which converges pointwise, locally uniformly everywhere to $\phi$ and $\left\|\phi_{m}\right\|_{\infty} \leq 2\|\phi\|_{\infty}$ for all $m \geq 1$. The convergence of $\rho_{n}(t, \cdot)$ to $\rho(t, \cdot)$ in the Wasserstein distance implies tightness, i.e. for any $\epsilon>0$ there exists $R>0$ such that

$$
\rho_{n}(t, \mathbb{R} \backslash(-R, R))<\epsilon \text { for all } n \geq 1 .
$$

Since

$$
\left|\int_{\mathbb{R}} v_{n}(t, y)\left[\phi(y)-\phi_{m}(y)\right] \rho_{n}(t, d y)\right| \leq\left\|v_{n}\right\|_{L^{2}\left(\rho_{n}(t, \cdot)\right)}\left\|\phi-\phi_{m}\right\|_{L^{2}\left(\rho_{n}(t, \cdot)\right)}
$$


and

$$
\left\|\phi-\phi_{m}\right\|_{L^{2}\left(\rho_{n}(t, \cdot)\right)}^{2}=\int_{-R}^{R}\left|\phi(y)-\phi_{m}(y)\right|^{2} \rho_{n}(t, d y)+\int_{\mathbb{R} \backslash(-R, R)}\left|\phi(y)-\phi_{m}(y)\right|^{2} \rho_{n}(t, d y),
$$

we deduce that $\left(\right.$ since $\left\|v_{n}(t, \cdot)\right\|_{L^{2}\left(\rho_{n}(t, \cdot)\right)} \leq\left\|v_{0}\right\|_{L^{2}\left(\rho_{0}\right)}$ by Proposition 3.2.1)

$$
\int_{\mathbb{R}} v_{n}(t, y)\left[\phi(y)-\phi_{m}(y)\right] \rho_{n}(t, d y) \underset{m \rightarrow \infty}{\longrightarrow} 0
$$

uniformly with respect to $n$. Likewise,

$$
\int_{\mathbb{R}} v(t, y)\left[\phi(y)-\phi_{m}(y)\right] \rho(t, d y) \underset{m \rightarrow \infty}{\longrightarrow} 0 .
$$

By

$$
\begin{aligned}
\int_{\mathbb{R}} v_{n}(t, y) \phi(y) \rho_{n}(t, d y) & -\int_{\mathbb{R}} v(t, y) \phi(y) \rho(t, d y)=\int_{\mathbb{R}} v_{n}(t, y)\left[\phi(y)-\phi_{m}(y)\right] \rho_{n}(t, d y) \\
& -\int_{\mathbb{R}} v(t, y)\left[\phi(y)-\phi_{m}(y)\right] \rho(t, d y) \\
& +\int_{\mathbb{R}} v_{n}(t, y) \phi_{m}(y) \rho_{n}(t, d y)-\int_{\mathbb{R}} v(t, y) \phi_{m}(y) \rho(t, d y),
\end{aligned}
$$

we infer the desired thesis.

Remark 3.2.1. Note that the method of proof of existence of the $S C L$ solution $(\rho, v)$ to (PEIC) with $\rho_{0} \in \mathcal{P}_{2}(\mathbb{R})$ and $v_{0} \in L^{2}\left(\rho_{0}\right)$ developed in [10] and [9] is essentially constructive. In summary, the steps of the construction are:

1. Approximate $\rho_{0} \in \mathcal{P}_{2}(\mathbb{R})$ by a sequence $\rho_{0, n}$ of finite summations of masses at discrete positions and denote by $M_{0, n}$ the right continuous cumulative distribution function of $\rho_{0, n}$.

2. Let $N_{0, n}$ be the optimal map pushing forward the Lebesgue measure on $(0,1)$ to $\rho_{0, n}$ and define the flux functions

$$
F_{n}(m):=\int_{0}^{m} v_{0} \circ N_{0, n}(x) d x .
$$

3. Produce a sequence of entropy solutions $M_{n}$ to $S C L-I C$.

4. Show that the entropy solutions $M_{n}$ converge to an entropy solution $M$ to SCL-IC.

5. Use $M$ to produce (as in Theorem 3.2.1 (ii), (iii)) the SCL solution ( $\rho, v)$ to (PE-IC) with initial conditions $\left(\rho_{0}, v_{0}\right)$. 


\subsection{Equivalence of SPF and SCL Solutions}

Here we first restrict $v_{0}$ to be essentially bounded with respect to $\rho_{0}$. Our goal is to show that if $v_{0}$ is right-continuous and bounded, then the SPF solution to (PE-IC) is unique and coincides with the SCL solution. We do so by incorporating and applying a third set of prior results [6] involving uniqueness of solutions for a similar system. The results which we wish to incorporate are:

Theorem 3.3.1. Let $\rho_{0} \geq 0 \in M_{\text {loc }}(\mathbb{R})$ (or $m_{0}(y)=\rho_{0}\left([0, y)\right.$ ) be non-decreasing) and $v_{0}$ be bounded and measurable to $\rho_{0}$. Let $\left(m_{1}, v_{1}\right)$ and $\left(m_{2}, v_{2}\right)$ be such that for $i \in\{1,2\}$,

1. for every $t \geq 0, m_{i}(t, \cdot)$ is of bounded variation locally,

2. $v_{i}(t, y)$ is bounded and measurable to $\rho_{i}=\partial_{y} m_{i}$,

3. the measures $\rho_{i}$ and $v_{i} \rho_{i}$ are weakly continuous in $t$,

4. for all $\phi \in C_{c}^{\infty}((0, \infty) \times \mathbb{R})$

$$
\int_{0}^{\infty} \int_{\mathbb{R}} \partial_{t} \phi(t, y) m_{i}(t, y) d y d t-\int_{0}^{\infty} \int_{\mathbb{R}} \phi(t, y) v_{i}(t, y) \rho_{i}(t, d y) d t=0,
$$

5. for all $\phi \in C_{c}^{\infty}((0, \infty) \times \mathbb{R})$

$$
\int_{0}^{\infty} \int_{\mathbb{R}}\left[\partial_{t} \phi(t, y) v_{i}(t, y)+\partial_{y} \phi(t, y) v_{i}^{2}(t, y)\right] \rho_{i}(t, d y) d t=0
$$

6. the measures $\rho_{i}(t, \cdot) v_{i}(t, \cdot)$ narrowly converge to $\rho_{0} v_{0}$ as $t \rightarrow 0^{+}$,

7. the measures $\rho_{i}(t, \cdot) v_{i}^{2}(t, \cdot)$ narrowly converge to $\rho_{0} v_{0}^{2}$ as $t \rightarrow 0^{+}$, i.e. (SICE) is satisfied,

8. for Lebesgue almost every $t \in(0, \infty)$

$$
\frac{v_{i}\left(y_{2}, t\right)-v_{i}\left(y_{1}, t\right)}{y_{2}-y_{1}} \leq \frac{1}{t} \text { for all } y_{1}<y_{2} \in \mathbb{R} .
$$

Then

$$
m_{1}=m_{2}
$$

and

$$
v_{1}=v_{2}
$$

a.e. with respect to the measure $\rho_{1}=\rho_{2}$.

We now state a corollary, which translates the above theorem in terms of right continuous cumulative distribution functions:

Corollary 3.3.1. Let $\rho_{0} \geq 0 \in M_{l o c}(\mathbb{R})$ (or $M_{0}(y)=\rho_{0}((-\infty, y])$ be non-decreasing) and $v_{0} \in L^{\infty}\left(\rho_{0}\right)$. Let $\left(M_{1}, v_{1}\right)$ and $\left(M_{2}, v_{2}\right)$ be such that for $i \in 1,2$,

1. for all $t \geq 0, M_{i}(t, \cdot)$ is of bounded variation locally, 
2. $v_{i}(t, y)$ is bounded and measurable to $\rho_{i}=\partial_{y} M_{i}$,

3. the measures $\rho_{i}$ and $v_{i} \rho_{i}$ are weakly continuous in $t$,

4. for all $\phi \in C_{c}^{\infty}((0, \infty) \times \mathbb{R})$

$$
\int_{0}^{\infty} \int_{\mathbb{R}} \partial_{t} \phi(t, y) M_{i}(t, y) d y d t-\int_{0}^{\infty} \int_{\mathbb{R}} \phi(t, y) v_{i}(t, y) \rho_{i}(t, d y) d t=0,
$$

5. for all $\phi \in C_{c}^{\infty}((0, \infty) \times \mathbb{R})$

$$
\int_{0}^{\infty} \int_{\mathbb{R}}\left[\partial_{t} \phi(t, y) v_{i}(t, y)+\partial_{y} \phi(t, y) v_{i}^{2}(t, y)\right] \rho_{i}(t, d y) d t=0,
$$

6. the measures $\rho_{i}(t, \cdot) v_{i}(t, \cdot)$ narrowly converge to $\rho_{0} v_{0}$ as $t \rightarrow 0^{+}$, i.e. $\left(\rho_{i}, v_{i}\right)$ satisfies (SICE),

7. the measure $\rho_{i}(t, \cdot) v_{i}^{2}(t, \cdot)$ narrowly converges to $\rho_{0} v_{0}^{2}$ as $t \rightarrow 0^{+}$,

8. condition (e-Oleinik) is satisfied by $v_{i}$.

Then

$$
M_{1}=M_{2}
$$

and

$$
v_{1}=v_{2}
$$

a.e. with respect to the measure $\rho_{1}=\rho_{2}$.

Proof. For $i \in\{1,2\}$ let

$$
m_{i}(t, y):=M_{i}(t, y)-\rho_{0}((-\infty, 0))-\rho_{i}(t,\{y\})
$$

we claim that $m_{i}$ satisfies the conditions of Theorem 3.3.1. Indeed, $\rho_{i}(t,\{y\})=0$ except for possibly at most countably many $y \in \mathbb{R}$, so $M_{i}(t, \cdot)-m_{i}(t, \cdot)=M_{0}(0)=$ const Lebesgue a.e. Thus, for almost every $t \in(0, \infty)$, the right continuous cumulative distribution functions $M_{1}(t, \cdot), M_{2}(t, \cdot)$ coincide everywhere.

\subsubsection{Preliminary Lemmas}

Prior to showing that SPF and SCL solutions coincide we need a few preliminary lemmas. The plan is to consider $v_{0}$ right-continuous and bounded (turns out that right-continuity is sufficient for ) and to show that if $(\rho, v)$ is either an SPF or the SCL solution to (PE-IC), then $(M, v)$ satisfies the conditions spelled out for $\left(M_{i}, v_{i}\right)$ in Corollary 3.3.1.

Lemma 3.3.1. Given $x_{1}, x_{2}, y_{1}, y_{2} \in \mathbb{R}$ with $x_{1}<x_{2}$, there exists a function $f \in C^{1}(\mathbb{R})$ such that the following properties hold:

1. $f(x)=y_{1}, \forall x \in\left(-\infty, x_{1}\right)$, 
2. $f(x)=y_{2}, \forall x \in\left(x_{2}, \infty\right)$,

3. $\left|f^{\prime}(x)\right| \leq 2 \frac{y_{2}-y_{1}}{x_{2}-x_{1}}, \forall x \in\left[x_{1}, x_{2}\right]$.

Proof. One candidate function can be defined by:

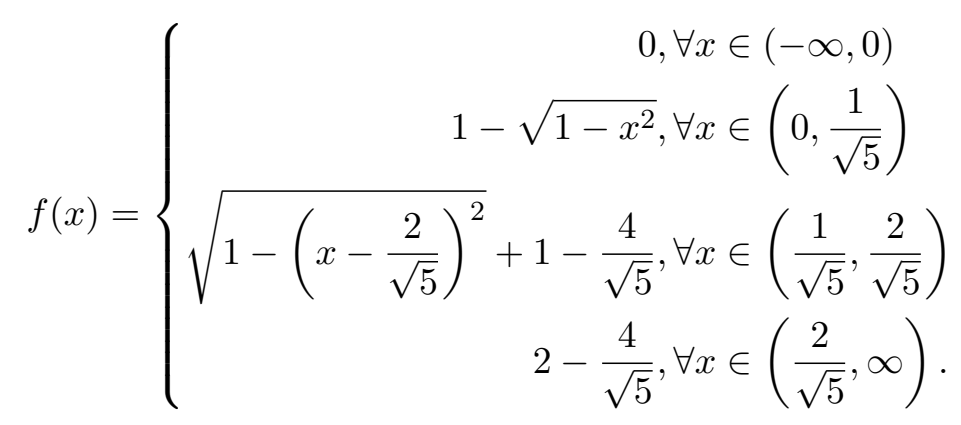

Note that $f \in C^{1}(\mathbb{R})$ and

1. $f(x)=0, \forall x \in(-\infty, 0)$.

2. $f(x)=2-\frac{4}{\sqrt{5}}, \forall x \in\left(\frac{2}{\sqrt{5}}, \infty\right)$.

3. $f^{\prime}(x)=0, \forall x \in(-\infty, 0)$.

4. $f^{\prime}(x)=0, \forall x \in\left(\left(\frac{2}{\sqrt{5}}, \infty\right)\right.$.

5. $\left|f^{\prime}(x)\right| \leq 2 \frac{f\left(\frac{2}{\sqrt{5}}\right)-f(0)}{\frac{2}{\sqrt{5}}-0}=2 \sqrt{5}-1, \forall x \in\left[0, \frac{2}{\sqrt{5}}\right]$.

6. $f\left(\frac{1}{\sqrt{5}}\right)=1-\frac{2}{\sqrt{5}}$.

7. $f^{\prime}\left(\frac{1}{\sqrt{5}}\right)=\frac{1}{2}$.

Using appropriate reflections, shifts, and scaling, it is obvious that this function can be altered to have the desired properties for any $x_{1}, x_{2}, y_{1}, y_{2} \in \mathbb{R}$ with $x_{1}<x_{2}$, not just for the specific example of $x_{1}=0, x_{2}=\frac{2}{\sqrt{5}}, y_{1}=0, y_{2}=2-\frac{4}{\sqrt{5}}$ as for this function. More concretely, the function $g$ defined as below satisfies the requirements:

$$
g(x)=y_{1}+\left(y_{2}-y_{1}\right) \frac{f\left(\frac{2\left(x-x_{1}\right)}{\sqrt{5}\left(x_{2}-x_{1}\right)}\right)}{2-\frac{4}{\sqrt{5}}} .
$$


Lemma 3.3.2. Suppose:

1. $\rho_{0} \in \mathcal{P}_{2}(\mathbb{R})$,

2. $\rho:[0, \infty) \rightarrow \mathcal{P}_{2}(\mathbb{R})$,

3. $v:[0, \infty) \times \mathbb{R} \rightarrow \mathbb{R}$ is Borel and satisfies $\int_{0}^{T}\|v(t, \cdot)\|_{L^{2}(\rho(t, \cdot))}^{2} d t<\infty$ for all $0<T<\infty$, are such that:

1. $\rho(t, \cdot)$ converges narrowly to $\rho_{0}$ and $v(t, \cdot) \rho(t, \cdot)$ converges narrowly to $v_{0} \rho_{0}$ as $t \rightarrow 0^{+}$,

2. $\forall \phi \in C_{c}^{\infty}((0, \infty) \times \mathbb{R})$ :

$$
\int_{0}^{\infty} \int_{\mathbb{R}}\left[\partial_{t} \phi+v \partial_{y} \phi\right] d \rho(t, \cdot) d t=0
$$

Then for any $\psi$ such that $\partial_{y} \psi \in C_{c}^{\infty}((0, \infty) \times \mathbb{R})$ :

$$
\int_{0}^{\infty} \int_{\mathbb{R}}\left[\partial_{t} \psi+v \partial_{y} \psi\right] d \rho(t, \cdot) d t=0
$$

Proof. Suppose $\rho_{0}, \rho, v$ are as in the assumptions. Suppose $\psi$ is such that $\partial_{y} \psi \in C_{c}^{\infty}((0, \infty) \times$ $\mathbb{R})$. Since $\partial_{y} \psi \in C_{c}^{\infty}((0, \infty) \times \mathbb{R})$, there exists $R>0$ such that $\partial_{y} \psi=0$ on $(0, \infty) \times(-\infty,-R) \cup$ $(R, \infty)$ and there exists $C_{1}, C_{2} \in \mathbb{R}$ such that $\psi(t, y)=C_{1}$ for all $(t, y) \in(0, \infty) \times(-\infty,-R]$ and $\psi(t, y)=C_{2}$ for all $(t, y) \in(0, \infty) \times[R, \infty)$. Using the previous lemma, let $f \in C^{1}(\mathbb{R})$ be defined such that the following properties hold:

1. $f(y)=0, \forall y \in\left(-\infty,-R-2 C_{1}\right)$,

2. $f(y)=C_{1}, \forall y \in(-R, \infty)$,

3. $\left|f^{\prime}(y)\right| \leq 1, \forall y \in\left[-R-2 C_{1},-R\right]$.

Also let $g \in C^{1}(\mathbb{R})$ be defined such that the following properties hold:

1. $g(y)=C_{2}, \forall y \in(-\infty, R)$,

2. $g(y)=0, \forall y \in\left(R+2 C_{2}, \infty\right)$,

3. $\left|g^{\prime}(y)\right| \leq 1, \forall y \in\left[R, R+2 C_{2}\right]$.

For all $m \in \mathbb{N}$ such that $m>R$, let $\psi_{m} \in C_{c}^{1}((0, \infty) \times \mathbb{R})$ be defined by:

$$
\psi_{m}(t, y):=\left\{\begin{array}{rr}
\psi(t, y) \text { if } & (t, y) \in(0, \infty) \times[-m, m] \\
f(y+m-R) \text { if } & (t, y) \in(0, \infty) \times(-\infty,-m) \\
g(y-m+R) \text { if } & (t, y) \in(0, \infty) \times(m, \infty) .
\end{array}\right.
$$


Note that $\psi_{m} \underset{m \rightarrow \infty}{\longrightarrow} \psi$ pointwise and that for all $(t, y) \in[0, \infty) \times \mathbb{R}$,

$$
\left|\psi(t, y)-\psi_{m}(t, y)\right| \leq \max \left\{\left|C_{1}\right|,\left|C_{2}\right|\right\}
$$

The Dominated Convergence Theorem (DCT) yields

$$
\int_{\mathbb{R}}\left|\psi(0, \cdot)-\psi_{m}(0, \cdot)\right| d \rho_{0} \underset{m \rightarrow \infty}{\longrightarrow} 0
$$

We now compute:

$$
\partial_{y} \psi_{m}(t, y):=\left\{\begin{array}{rr}
\partial_{y} \psi(t, y) \text { if } & (t, y) \in(0, \infty) \times[-m, m] \\
f^{\prime}(y+m-R) \text { if } & (t, y) \in(0, \infty) \times(-\infty,-m) \\
g^{\prime}(y-m+R) \text { if } & (t, y) \in(0, \infty) \times(m, \infty) .
\end{array}\right.
$$

Note that $\partial_{y} \psi_{m} \underset{m \rightarrow \infty}{\longrightarrow} \partial_{y} \psi$ pointwise everywhere, so $v \partial_{y} \psi_{m} \underset{m \rightarrow \infty}{\longrightarrow} v \partial_{y} \psi$ pointwise, $\rho(t, \cdot)$ almost everywhere. Also,

$$
\forall(t, y) \in(0, \infty) \times \mathbb{R},\left|v(t, y) \partial_{y} \psi(t, y)-v(t, y) \partial_{y} \psi_{m}(t, y)\right| \leq|v(t, y)|, \text { for } \rho(t, \cdot)-\text { a.e.y. }
$$

Also note that $v \in L^{2}(\rho(t, \cdot))$ and since $\rho(t, \cdot)$ is a probability measure, this implies $v \in$ $L^{1}(\rho(t, \cdot))$. Thus, by DCT,

$$
\int_{\mathbb{R}}\left|v(t, y) \partial_{y} \psi(t, y)-v(t, y) \partial_{y} \psi_{m}(t, y)\right| \rho(t, d y) \underset{m \rightarrow \infty}{\longrightarrow} 0
$$

Finally, we compute:

$$
\partial_{t} \psi_{m}(t, y):=\left\{\begin{array}{rrr}
\partial_{t} \psi(t, y) \text { if } & (t, y) \in(0, \infty) \times[-m, m] \\
0 \text { if } & (t, y) \in(0, \infty) \times(-\infty,-m) \\
0 \text { if } & (t, y) \in(0, \infty) \times(m, \infty) .
\end{array}\right.
$$

Note that $\partial_{t} \psi_{m} \underset{m \rightarrow \infty}{\longrightarrow} \partial_{t} \psi$ pointwise everywhere, and that $\forall(t, y) \in(0, \infty) \times \mathbb{R}, \mid \partial_{t} \psi(t, y)-$ $\partial_{t} \psi_{m}(t, y) \mid \leq \max \left\{\left|\partial_{t} \psi\right|\right\}<\infty$ since there exists $T>0$ such that $\partial_{t} \psi(t, y)=0$ for all $t>T$. By DCT, we get

$$
\int_{\mathbb{R}}\left|\partial_{t} \psi(t, y)-\partial_{t} \psi_{m}(t, y)\right| \rho(t, d y) \underset{m \rightarrow \infty}{\longrightarrow} 0
$$

Finally, as $\psi_{m} \in C_{c}^{1}((0, \infty) \times \mathbb{R})$, we have

$$
\int_{0}^{\infty} \int_{\mathbb{R}}\left[\partial_{t} \psi_{m}+v \partial_{y} \psi_{m}\right] d \rho(t, \cdot) d t=0 .
$$


Using these facts, we have that, for $\psi$ such that $\partial_{y} \psi \in C_{c}^{\infty}((0, \infty) \times \mathbb{R})$ and for $m>R$ :

$$
\begin{aligned}
\left|\int_{0}^{\infty} \int_{\mathbb{R}}\left[\partial_{t} \psi+v \partial_{y} \psi\right] d \rho(t, \cdot) d t\right| & \mid \int_{0}^{\infty} \int_{\mathbb{R}}\left[\left(\partial_{t} \psi-\partial_{t} \psi_{m}\right)+v\left(\partial_{y} \psi-\partial_{y} \psi_{m}\right)\right] d \rho(t, \cdot) d t \\
& +\int_{0}^{\infty} \int_{\mathbb{R}}\left[\partial_{t} \psi_{m}+v \partial_{y} \psi_{m}\right] d \rho(t, \cdot) d t \mid \\
= & \mid \int_{0}^{\infty} \int_{\mathbb{R}}\left[\left(\partial_{t} \psi-\partial_{t} \psi_{m}\right)+v\left(\partial_{y} \psi-\partial_{y} \psi_{m}\right)\right] d \rho(t, \cdot) d t \\
& \leq \int_{0}^{\infty} \int_{\mathbb{R}}\left|\partial_{t} \psi-\partial_{t} \psi_{m}\right| d \rho(t, \cdot) d t \\
& +\int_{0}^{\infty} \int_{\mathbb{R}}\left|v\left(\partial_{y} \psi-\partial_{y} \psi_{m}\right)\right| d \rho(t, \cdot) d t .
\end{aligned}
$$

Earlier, we showed that the terms on the right hand side go to 0 as $m \rightarrow \infty$. Thus

$$
\int_{0}^{\infty} \int_{\mathbb{R}}\left[\partial_{t} \psi+v \partial_{y} \psi\right] d \rho(t, \cdot) d t=0
$$

\subsubsection{From (Oleinik) to (e-Oleinik)}

Here we show that if $v_{0}$ is right-continuous and bounded, then any SPF solution and the SCL solution to (PE-IC) satisfy the following:

Proposition 3.3.1. Let $\rho_{0} \in \mathcal{P}_{2}(\mathbb{R})$ and $v_{0}: \mathbb{R} \rightarrow \mathbb{R}$ be right-continuous and bounded. Let $(\rho, v)$ be an SPF or the SCL solution to $(P E-I C)$. Then there exists a Borel map $\hat{v}:(0, \infty) \times$ $\mathbb{R} \rightarrow \mathbb{R}$ such that $\hat{v}(t, \cdot)=v(t, \cdot) \rho(t, \cdot)$-a.e. and $\hat{v}$ satisfies (e-Oleinik) and $|\hat{v}| \leq\left\|v_{0}\right\|_{L^{\infty}(\mathbb{R})}$ on the whole $(0, \infty) \times \mathbb{R}$.

Before we prove this let us observe that (e-Oleinik) is stronger than (Oleinik) because the inequality

$$
\frac{v\left(t, y_{2}\right)-v\left(t, y_{1}\right)}{y_{2}-y_{1}} \leq \frac{1}{t}
$$

must be satisfied for every $y_{1}<y_{2} \in \mathbb{R}$ (and not just in the $\rho(t, \cdot)$-a.e. sense). The proof of Proposition 3.3.1 will be based on a structure result for the velocity $v$.

Lemma 3.3.3. Let $\rho_{0} \in \mathcal{P}_{2}(\mathbb{R})$ and $v_{0} \in L^{2}\left(\rho_{0}\right)$. Let $(\rho, v)$ be an $S P F$ or the $S C L$ solution to $(P E-I C)$. For $t \geq 0$ denote by $N(t, \cdot)$ the right-continuous optimal map pushing $\left.\mathcal{L}^{1}\right|_{I}$ forward to $\rho(t, \cdot)$, where $I:=(0,1)$, and let $V_{0}:=v_{0} \circ N_{0}$. Let $\bar{v}(t, \cdot)$ defined on $N(t, I)$ by

$$
\bar{v}(t, y)= \begin{cases}V_{0} \circ M(t, y) & \text { if }[N(t, \cdot)]^{-1}(y) \text { is a singleton } \\ f_{M(t, y-)}^{M(t, y)} V_{0}(s) d s & \text { if }[N(t, \cdot)]^{-1}(y) \text { is not a singleton. }\end{cases}
$$


Then the following hold:

(i) For all $t \geq 0$ we have $v(t, \cdot)=\bar{v}(t, \cdot) \rho(t, \cdot)$-a.e.

(ii) Moreover, if $v_{0} \in C_{r}(\mathbb{R})$ (right-continuous on $\mathbb{R}$ ) and $v$ satisfies (Oleinik), then $\bar{v}$ satisfies (e-Oleinik) on the image of $N$.

Proof. 1. Since $N_{t}:=N(t, \cdot)$ pushes $\left.\mathcal{L}^{1}\right|_{I}$ forward to $\rho_{t}$, we have

$$
\rho_{t}\left(N_{t}(I)\right)=\mathcal{L}^{1}\left(N_{t}^{-1}\left(N_{t}(I)\right)\right) \geq \mathcal{L}^{1}(I)=1,
$$

so $\rho_{t}\left(N_{t}(I)\right)=1$. Now, if $(\rho, v)$ is an SPF solution, we use Definition 1.3.5 and (1.3.1), along with $N_{t}=X_{t} \circ N_{0}$, to write

$$
\begin{aligned}
\int_{\mathbb{R}} v_{t}(y) \phi(y) \rho_{t}(d y) & =\int_{0}^{1} v_{t}\left(N_{t}(x)\right) \phi\left(N_{t}(x)\right) d x \\
& =\int_{0}^{1} V_{0}(x) \phi\left(N_{t}(x)\right) d x \text { for all } \phi \in C_{b}(\mathbb{R}) .
\end{aligned}
$$

Next, we disintegrate $\left.\mathcal{L}^{1}\right|_{I}$ with respect to the level sets of the optimal push forward map $N_{t}$ to get

$$
\int_{0}^{1} V_{0}(x) \phi\left(N_{t}(x)\right) d x=\int_{\mathbb{R}} \phi(y)\left[\int_{N_{t}^{-1}(y)} V_{0}(z) \nu_{t, y}(d z)\right] \rho_{t}(d y),
$$

where $\nu_{t, y}$ are Borel probability measures on the level set $N_{t}^{-1}(y)$. It follows that

$$
v_{t}(y)=\int_{N_{t}^{-1}(y)} V_{0}(z) \nu_{t, y}(d z) \text { for } \rho_{t} \text {-a.e. } y \in \mathbb{R} .
$$

Since $N_{t}$ is non-decreasing, $N_{t}^{-1}(y)$ is either empty, a singleton (in which case, $N_{t}^{-1}(y)=$ $M_{t}(y)=M_{t}(y-)$ ) or a non-degenerate subinterval of $I$. Furthermore, by Theorem 3.16 in [5], $\nu_{t, y}=\delta_{N_{t}^{-1}(y)}$ if $N_{t}^{-1}(y)$ is a singleton, and $N_{t}^{-1}(y)$ is the uniform probability distribution on the interval $N_{t}^{-1}(y)$ if $N_{t}^{-1}(y)$ is a non-degenerate interval (i.e. the normalized Lebesgue measure on said interval). Since $M$ and $N$ are non-decreasing and generalized inverses of each other, i.e.

$$
M_{t}(y)=\inf \left\{x \in I: N_{t}(x)>y\right\}, N_{t}(x)=\inf \left\{y \in \mathbb{R}: M_{t}(y)>x\right\}
$$

we see that $N_{t}^{-1}(y)$ has endpoints $M_{t}(y-)$ and $M_{t}(y)$ whenever it is a non-degenerate interval (i.e. $\left.\rho_{t}(\{y\})=M_{t}(y)-M_{t}(y-)\right)$. So, the proposition is proved for SPF solutions.

If we consider a sequence of smooth, strictly positive probability densities $\rho^{\epsilon}$ which converges in $W_{2}$ to $\rho_{t}$, we have that $M^{\epsilon}-M_{t}$ converges to zero in $L^{1}(\mathbb{R})$ and $N^{\epsilon}$ converges to $N_{t}$ in $L^{2}(I)$, due to

$$
\left\|N^{\epsilon}-N_{t}\right\|_{L^{2}(I)}=W_{2}\left(\rho^{\epsilon}, \rho_{t}\right) \geq W_{1}\left(\rho^{\epsilon}, \rho_{t}\right)=\left\|M^{\epsilon}-M_{t}\right\|_{L^{1}(\mathbb{R})} .
$$


We have

$$
\begin{aligned}
\int_{\mathbb{R}} \phi^{\prime}(y) F(M(t, y)) d y & =\lim _{\epsilon \rightarrow 0^{+}} \int_{\mathbb{R}} \phi^{\prime}(y) F\left(M^{\epsilon}(y)\right) d y \\
& =-\lim _{\epsilon \rightarrow 0^{+}} \int_{\mathbb{R}} \phi(y) V_{0}\left(M^{\epsilon}(y)\right) \rho^{\epsilon}(y) d y \\
& =-\lim _{\epsilon \rightarrow 0^{+}} \int_{0}^{1} \phi\left(N^{\epsilon}(x)\right) V_{0}(x) d x \\
& =-\int_{0}^{1} V_{0}(x) \phi\left(N_{t}(x)\right) d x \text { for all } \phi \in C_{c}^{1}(\mathbb{R}) .
\end{aligned}
$$

But (3.2.4) holds for SCL solutions, so (3.3.2) holds for SCL solutions as well, albeit the test functions are now restricted to $C_{c}^{1}(\mathbb{R})$. This restriction, obviously, does not prevent us from drawing the same conclusion, so (i) is proved.

2. Let $t>0$. For all $x \in I$, denote by $l_{t}(x):=\inf \left\{z \in I: N_{t}(z)=N_{t}(x)\right\}$ and $r_{t}(x):=$ $\sup \left\{z \in I: N_{t}(z)=N_{t}(x)\right\}$; clearly, $0 \leq l_{t}(x) \leq x \leq r_{t}(x) \leq 1$ for all $x \in I$. Assume $N_{t}$ is not constant on $I$ (in which case, there is nothing to prove). Since $\rho_{t}\left(N_{t}(I)\right)=1$, (Oleinik) is equivalent to the existence of a subset $A \subset N_{t}(I)$ such that $\rho_{t}(A)=1$ and the map $y \rightarrow y-t \bar{v}(t, y)$ is nondecreasing on $A$. This implies $x \rightarrow N_{t}(x)-t \bar{v}\left(t, N_{t}(x)\right)=: w_{t}(x)$ is nondecreasing on $N_{t}^{-1}(A)=: J$, which is a subset of $I$ of unit Lebesgue measure (since $\left.\mathcal{L}^{1}(J)=\rho_{t}(A)\right)$. Let $0<a<b<1$.

Case 1: If $N_{t}^{-1}\left(N_{t}(a)\right) \neq\{a\}$, then the former is a nondegenerate interval, so it contains some $x \in J$; it follows $N_{t}(a)=N_{t}(x) \in A$, and so $a \in J$. If $b \in J$, we get $w_{t}(a) \leq w_{t}(b)$ because $w_{t}$ is nondecreasing on $J$. If $b \notin J$, then $N_{t}(a)<N_{t}(b)$ and $N_{t}^{-1}\left(N_{t}(b)\right)=\{b\}$ (otherwise, just as with $a$, we deduce $b \in J)$. Since $w_{t}$ is nondecreasing on $J$, we have

$$
w_{t}(a) \leq w_{t}\left(x_{n}\right)=N_{t}\left(x_{n}\right)-t f_{l_{t}\left(x_{n}\right)}^{r_{t}\left(x_{n}\right)} V_{0}(s) d s
$$

for any decreasing sequence $\left\{x_{n}\right\}_{n} \subset(b, 1) \cap J$ which converges to $b$ (the average is interpreted as $V_{0}(x)$ if $l_{t}(x)=x=r_{t}(x)$ ). Since $l_{t}(b)=r_{t}(b)=b$ (so $N_{t}$ is not constant in any interval of the type $[b, b+\delta)$ ) we deduce $r_{t}\left(x_{n}\right)-l_{t}\left(x_{n}\right) \rightarrow 0$ as $n \rightarrow \infty$. Indeed, note that $r_{t}(x)-$ $l_{t}(x)=\rho_{t}\left(\left\{N_{t}(x)\right\}\right)$, and since $N_{t}\left(x_{n}\right)>N_{t}(b)$ for all $n \geq 1$ (because $N_{t}^{-1}\left(N_{t}(b)\right)=\{b\}$ ) and $N_{t}\left(x_{n}\right) \rightarrow N_{t}(b)+$, we deduce that the sequence $\left\{N_{t}\left(x_{n}\right)\right\}_{n}$ does not become stationary; so $\rho_{t}\left(\left\{N_{t}\left(x_{n}\right)\right\}\right)$ must converge to zero (as $\rho_{t}$ has finite total mass). But $b<l_{t}\left(x_{n}\right) \leq x_{n} \leq r_{t}\left(x_{n}\right)$, so the right continuity of $V_{0}$ (as the composition of the right-continuous $v_{0}$ with the rightcontinuous and nondecreasing $N_{0}$ ) implies, in view of the right continuity of $N_{t}$ and the displayed inequality above,

$$
w_{t}(a) \leq N_{t}(b)-t V_{0}(b)=N_{t}(b)-t V_{0}\left(M_{t}\left(N_{t}(b)\right)=w_{t}(b) .\right.
$$

Case 2: Now assume $N_{t}^{-1}\left(N_{t}(a)\right)=\{a\}$. If $a \in J$, we conclude exactly as in Case 1 above. If $a \notin J$, let $\left\{x_{n}\right\}_{n} \subset J \cap(a, b)$ be a decreasing sequence which converges to $a$. If $b \in J$ we have $w_{t}\left(x_{n}\right) \leq w_{t}(b)$. Since $l_{t}(a)=a=r_{t}(a)$, we deduce (as above) $r_{t}\left(x_{n}\right)-l_{t}\left(x_{n}\right) \rightarrow 0$ as $n \rightarrow \infty$ and so, again, in the limit we get

$$
w_{t}(a)=N_{t}(a)-t V_{0}(a)=\lim _{n \rightarrow \infty}\left[N_{t}\left(x_{n}\right)-t f_{l_{t}\left(x_{n}\right)}^{r_{t}\left(x_{n}\right)} V_{0}(s) d s\right]=\lim _{n \rightarrow \infty} w_{t}\left(x_{n}\right) \leq w_{t}(b) .
$$


If $b \notin J$, we have (again, as above) that necessarily $N_{t}^{-1}\left(N_{t}(b)\right)=\{b\}$. So we now consider, beside the sequence $\left\{x_{n}\right\}_{n}$, another sequence $\left\{z_{n}\right\}_{n} \subset(b, 1) \cap J$ which is decreasing and converges to $b$. Since $x_{n}<z_{n}$ and $x_{n}, z_{n} \in J$, we have

$w_{t}(a)=\lim _{n \rightarrow \infty}\left[N_{t}\left(x_{n}\right)-t f_{l_{t}\left(x_{n}\right)}^{r_{t}\left(x_{n}\right)} V_{0}(s) d s\right] \leq \lim _{n \rightarrow \infty}\left[N_{t}\left(z_{n}\right)-t f_{l_{t}\left(z_{n}\right)}^{r_{t}\left(z_{n}\right)} V_{0}(s) d s\right]=\lim _{n \rightarrow \infty} w_{t}\left(z_{n}\right)=w_{t}(b)$.

This finishes the proof of (ii).

Remark 3.3.1. We now have a map $\bar{v}$ defined everywhere on the set (a Borel set as the union of $\mathcal{R}$ and $\mathcal{D}$; see Theorem 5.2.2 in Appendix)

$$
\mathcal{N}:=\bigcup_{t \in[0, \infty)}\{t\} \times N_{t}(I)
$$

and also $\rho_{t}\left(N_{t}(I)\right)=1$, so $v(t, \cdot)=\bar{v}(t, \cdot) \rho_{t}$-a.e. Note that, by $(3.3 .1)$, if $\rho_{0} \in \mathcal{P}_{2}(\mathbb{R})$ and $v_{0}: \mathbb{R} \rightarrow \mathbb{R}$ is a bounded Borel map, then $|\bar{v}(t, y)| \leq\left\|v_{0}\right\|_{L^{\infty}(\mathbb{R})}$ for all $(t, y) \in \mathcal{N}$. Next we show that if $v_{0}$ is also right-continuous, then, under the assumption (Oleinik) on $v$ (or, equivalently, on $\bar{v})$, there exists a Borel map $\hat{v}$ defined on the whole $[0, \infty) \times \mathbb{R}$, which satisfies (e-Oleinik) on $(0, \infty) \times \mathbb{R}, \hat{v}=\bar{v}$ on $\mathcal{N}$ and $|\hat{v}| \leq\left\|v_{0}\right\|_{L^{\infty}(\mathbb{R})}$ on $[0, \infty) \times \mathbb{R}$.

Proof. (of Proposition 3.3.1) Showing existence of $\hat{v}$ will be done by construction, the first step of which is to partition $[0, \infty) \times \mathbb{R}$ into the Borel sets $\mathcal{Z}, \mathcal{J}, \mathcal{S}, \mathcal{O}, \mathcal{D}, \mathcal{R}, \mathcal{U}$ defined in the statement of Proposition 5.2.2 (Appendix). Let $l, r$ be the Borel maps defined in the statement of Proposition 5.2.1 (Appendix). Since Lemma 3.3.3 defines $\bar{v}$ everywhere in $\mathcal{N}:=\mathcal{D} \cup \mathcal{R}$, all we need is to provide an appropriate (should be a Borel function with the explicit bound discussed in Remark 3.3.1) explicit extension to $\mathcal{Z} \cup \mathcal{J}, \mathcal{S} \cup \mathcal{O}$ and $\mathcal{U}$. By Proposition 5.2.3, we see that for each $t>0$, the fiber $\mathcal{U}_{t}$ of $\mathcal{U}$ is the union of all the intervals $[N(t, x-), N(t, x))$ for all points $x \in I$ where $N(t, \cdot)$ is discontinuous. Since $y \rightarrow y-t \bar{v}(t, y)$ is nondecreasing on $N(t, I)$, we deduce that

$$
\lim _{z \rightarrow x^{-}}[N(t, z)-t \bar{v}(t, N(t, z))] \text { exists in } \mathbb{R}
$$

but $N(t, x-) \in \mathbb{R}$ exists as well, so the $\operatorname{limit}_{\lim _{z \rightarrow x^{-}}} \bar{v}(t, N(t, z))$ exists in $\mathbb{R}$. We assign this limit as the value of $\hat{v}$ at $y=N(t, x-)=l(t, y)$ for all points of discontinuity $x$ of $N(t, \cdot)$. To translate this in terms of $(t, y)$, according to Proposition 5.2.3 (5), we conclude

$$
\left.\hat{v}(t, y)=\lim _{z \rightarrow y^{-}, z \in N(t, I)} \bar{v}(t, z)=\lim _{n \rightarrow \infty} \bar{v}(t, N(t, x-1 / n))\right)=: \bar{v}(t, l(t, y)-)
$$

for all $(t, y) \in \mathcal{M} \cap(\mathcal{A} \backslash \mathcal{D})$. Since this is a Borel set (due to Proposition 5.2.2) and $l, N, \bar{v}$ are Borel functions (due to Propositions 5.2.1, 5.2.2), we deduce that this extension $\hat{v}$ (from $\mathcal{D} \cup \mathcal{R}$ to $\mathcal{D} \cup \mathcal{R} \cup[\mathcal{M} \cap(\mathcal{A} \backslash \mathcal{D})])$ is Borel on $\mathcal{D} \cup \mathcal{R} \cup[\mathcal{M} \cap(\mathcal{A} \backslash \mathcal{D})]$. So now $\hat{v}$ is defined at all points $N(t, x)$ and $N(t, x-), x \in I$. Since $y \rightarrow y-t \bar{v}(t, y)$ is nondecreasing on $\mathcal{D} \cup \mathcal{R}$ (i.e. $\bar{v}$ satisfies (e-Oleinik) on this set) and the extension is defined by using left-sided limits along points in $\mathcal{D} \cup \mathcal{R}$, we deduce $y \rightarrow y-t \hat{v}(t, y)$ is nondecreasing (for fixed $t>0$ ) on $\mathcal{D} \cup \mathcal{R} \cup[\mathcal{M} \cap(\mathcal{A} \backslash \mathcal{D})]$, i.e. $\hat{v}$ satisfies (e-Oleinik) there. Next we extend $\hat{v}(t, \cdot)$ to the intervals $(N(t, x-), N(t, x))$ by interpolating linearly between the values at $N(t, x-)$ and $N(t, x)$. Let 
$N(t, x-)<y<N(t, x)$ for some $x$ in the discontinuity set of $N(t, \cdot)$; then $l(t, y)=N(t, x-)$ and $r(t, y)=N(t, x)$. We have $\hat{v}(t, r(t, y))$ and $\hat{v}(t, l(t, y))$ already defined above (because $r(t, y) \in N(t, I)$ and $l(t, y) \in \mathcal{M} \cap(\mathcal{A} \backslash \mathcal{D})$; now set

$$
\hat{v}(t, y):=\frac{r(t, y)-y}{r(t, y)-l(t, y)} \bar{v}(t, l(t, y)-)+\frac{y-l(t, y)}{r(t, y)-l(t, y)} \bar{v}(t, r(t, y))
$$

which is equivalent to

$$
w(t, y):=\frac{r(t, y)-y}{r(t, y)-l(t, y)} w(t, l(t, y))+\frac{y-l(t, y)}{r(t, y)-l(t, y)} w(t, r(t, y))
$$

where $w(t, y):=y-t \hat{v}(t, y)$. Since $w(t, \cdot)$ is nondecreasing on $\mathcal{D} \cup \mathcal{R} \cup[\mathcal{M} \cap(\mathcal{A} \backslash \mathcal{D})]$ and on the gap interval $(l(t, y), r(t, y))$ we have interpolated linearly, it follows that $w(t, \cdot)$ is nondecreasing as a function of $y$ everywhere on $\mathcal{M}$, i.e. $w(t, \cdot)$ is nondecreasing on $(N(t, 0+), N(t, 1-))$. On $(-\infty, N(t, 0+)]$ (if $N(t, 0+)>-\infty$ ) we let $w(t, y):=y-t\left\|v_{0}\right\|_{L^{\infty}(\mathbb{R})}$, whereas on $[N(t, 1-), \infty)$ (if $N(t, 1-)<\infty$ ) we let $w(t, y):=y+t\left\|v_{0}\right\|_{L^{\infty}(\mathbb{R})}$; it is easy (by using the above monotonicity and the bound on $\left.v_{0}\right)$ to see that $w(t, \cdot)$ is nondecreasing on the whole $\mathbb{R}$.

To summarize, we have defined $\hat{v}$ as follows:

$$
\hat{v}(t, y):=\left\{\begin{array}{rrr}
\frac{r(t, y)-y}{r(t, y)-l(t, y)} \bar{v}(t, l(t, y)-)+\frac{y-l(t, y)}{r(t, y)-l(t, y)} \bar{v}(t, r(t, y)) \text { if } & (t, y) \in \mathcal{U} \\
\left\|v_{0}\right\|_{L^{\infty}(\mathbb{R})} \text { if } & (t, y) \in \mathcal{Z} \cup \mathcal{J} \\
-\left\|v_{0}\right\|_{L^{\infty}(\mathbb{R})} \text { if } & (t, y) \in \mathcal{S} \cup \mathcal{O},
\end{array}\right.
$$

where $\bar{v}$ is defined in (3.3.1) and $\bar{v}(t, l(t, \cdot)-)$ is defined by (3.3.3). The fact that $w(t, \cdot)$ is nondecreasing on $\mathbb{R}$ is equivalent to $\hat{v}$ satisfying (e-Oleinik) on $(0, \infty) \times \mathbb{R}$. We also see that $|\hat{v}| \leq\left\|v_{0}\right\|_{L^{\infty}(\mathbb{R})}$ on $\mathbb{R}$.

\subsubsection{SPF solution satisfies Corollary 3.3.1 assumptions}

We now show that the SPF solution $(\rho, v)$ yields a pair $(M, v)$ satisfying the conditions spelled out in Corollary 3.3.1.

Proposition 3.3.2. Suppose $\rho_{0} \in \mathcal{P}_{2}(\mathbb{R})$ and $v_{0}: \mathbb{R} \rightarrow \mathbb{R}$ be right-continuous and bounded. Let $(\rho, v)$ be an SPF solution to $(P E-I C)$. Let $M(t, y)=\rho(t,(-\infty, y])$ be the cumulative distribution function for $\rho(t, \cdot)$. Then there exists a Borel map $\hat{v}:[0, \infty) \times \mathbb{R}$ such that for all $t \geq 0$ we have $\hat{v}(t, \cdot)=v(t, \cdot) \rho(t, \cdot)$-a.e. and $(M, \hat{v})$ satisfies the conditions of Corollary 3.3.1.

Proof. (1) is obvious since $M(t, \cdot)$ is the cumulative distribution function of the probability measure $\rho(t, \cdot)$. Note that $(3),(5),(6),(7)$ follow from Proposition 3.1.1, while (8) is a consequence of Proposition 3.3.1. Thus, we only need to show (2), (4).

(2) The definition of the conditional expectation yields

$$
I(\phi):=\int_{\mathbb{R}} v(t, y) \phi(y) \rho(t, d y)=\int_{\mathbb{R}} v_{0}(y) \phi(X(t, y)) \rho_{0}(d y) .
$$


It follows

$$
\begin{aligned}
|I(\phi)|=\left|\int_{\mathbb{R}} v_{0}(y) \phi(X(t, y)) \rho_{0}(d y)\right|=\left\|v_{0} \cdot\left(\phi \circ X_{t}\right)\right\|_{L^{1}\left(\rho_{0}\right)} & \leq\left\|v_{0}\right\|_{L^{\infty}\left(\rho_{0}\right)}\left\|\phi \circ X_{t}\right\|_{L^{1}\left(\rho_{0}\right)} \\
& =\left\|v_{0}\right\|_{L^{\infty}\left(\rho_{0}\right)}\|\phi\|_{L^{1}\left(\rho_{t}\right)} .
\end{aligned}
$$

Thus, $I$ is a bounded linear functional on $L^{1}\left(\rho_{t}\right)$ of norm at most $\left\|v_{0}\right\|_{L^{\infty}\left(\rho_{0}\right)}$, so $v_{t} \in L^{\infty}\left(\rho_{t}\right)$ and $\left\|v_{t}\right\|_{L^{\infty}\left(\rho_{t}\right)} \leq\left\|v_{0}\right\|_{L^{\infty}\left(\rho_{0}\right)}$.

(4) What we need to show is that for all $\phi \in C_{c}^{\infty}((0, \infty) \times \mathbb{R})$

$$
\int_{0}^{\infty} \int_{\mathbb{R}} \partial_{t} \phi(t, y) M(t, y) d y d t-\int_{0}^{\infty} \int_{\mathbb{R}} v(t, y) \phi(t, y) \rho(t, d y) d t=0 .
$$

So suppose $\phi \in C_{c}^{\infty}((0, \infty) \times \mathbb{R})$. Now define $\psi:(0, \infty) \times \mathbb{R} \rightarrow \mathbb{R}$ by

$$
\psi(t, y):=\int_{-\infty}^{y} \phi(t, z) d z .
$$

Note that $\psi$ is compactly supported in $(0, \infty)$ in time. Now, we have

$$
\begin{aligned}
\int_{\mathbb{R}} \partial_{t} \psi(t, y) \rho(t, d y) & =\left.M(t, y) \partial_{t} \psi(t, y)\right|_{y=-\infty} ^{y=\infty}-\int_{\mathbb{R}} \partial_{y} \partial_{t} \psi(t, y) M(t, y) d y \\
& =\partial_{t} \psi(t, \infty)-\int_{\mathbb{R}} \partial_{t} \phi(t, y) M(t, y) d y
\end{aligned}
$$

where we have taken into account the fact that $M(t, \cdot)$ is a probability cumulative distribution function and $\psi(t, \infty)=\int_{\mathbb{R}} \phi(t, y) d y$. Then, since $\partial_{y} \psi=\phi \in C_{c}^{\infty}((0, \infty) \times \mathbb{R})$, by Lemma 3.3.2, we have that

$$
\begin{aligned}
0 & =\int_{0}^{\infty} \int_{\mathbb{R}}\left[\partial_{t} \psi(t, y)+v(t, y) \partial_{y} \psi(t, y)\right] \rho(t, d y) d t \\
& =\int_{0}^{\infty} \int_{\mathbb{R}} \partial_{t} \psi(t, y) \rho(t, d y) d t+\int_{0}^{\infty} \int_{\mathbb{R}} v(t, y) \partial_{y} \psi(t, y) \rho(t, d y) d t \\
& =-\int_{0}^{\infty} \int_{\mathbb{R}} \partial_{t} \partial_{y} \psi(t, y) M(t, y) d y d t+\int_{0}^{\infty} \int_{\mathbb{R}} v(t, y) \partial_{y} \psi(t, y) \rho(t, d y) d t \\
& =-\int_{0}^{\infty} \int_{\mathbb{R}} \partial_{t} \phi(t, y) M(t, y) d y d t+\int_{0}^{\infty} \int_{\mathbb{R}} v(t, y) \phi(t, y) \rho(t, d y) d t
\end{aligned}
$$

(8) We know from Theorem 3.1.1 that $(\rho, v)$ satisfies the Oleinik condition (Oleinik). By Theorem 3.3.1, we have that $v$ admits a Borel representative (in the sense described there) $\hat{v}$ which satisfies (e-Oleinik).

\subsubsection{SCL solution satisfies Corollary 3.3.1 assumptions}

Now we show that the scalar conservation law characterization satisfies the 3.3.1 notion of solution. 
Proposition 3.3.3. Suppose $\rho_{0} \in \mathcal{P}_{2}(\mathbb{R})$ and $v_{0}: \mathbb{R} \rightarrow \mathbb{R}$ be right-continuous and bounded. Let $(\rho, v)$ be the $S C L$ solution to $(P E-I C)$. Let $M(t, y)=\rho(t,(-\infty, y])$ be the cumulative distribution function of $\rho(t, \cdot)$. Then there exists a Borel map $\hat{v}:[0, \infty) \times \mathbb{R}$ such that for all $t \geq 0$ we have $\hat{v}(t, \cdot)=v(t, \cdot) \rho(t, \cdot)$-a.e. and $(M, \hat{v})$ satisfies the conditions of Corollary 3.3.1.

Proof. Just as in the proof of Proposition 3.3.2, we skip the straight-forward parts and focus on the others.

(3) (Proof that $v$ is bounded with respect to $\rho=\partial_{y} M$ ) Note that in the development in [10], the flux function $F$ was defined as:

$$
F(m)=\int_{0}^{m} v_{0} \circ N_{0}(x) d x
$$

In addition, $F(M(t, \cdot))$ has spatial distributional derivative $v(t, \cdot) \rho(t, \cdot)$, thus for all $\phi \in C_{c}^{1}(\mathbb{R})$ :

$$
\int_{\mathbb{R}} v(t, y) \phi(y) \rho(t, d y)=-\int_{\mathbb{R}} F(M(t, y)) \phi^{\prime}(y) \rho(t, d y)=\int_{\mathbb{R}} F^{\prime}(M(t, y)) \phi(y) \rho(t, d y) .
$$

Since $v_{0} \in L^{\infty}\left(\rho_{0}\right)$, we get

$$
\begin{aligned}
\left|\int_{\mathbb{R}} v(t, y) \phi(y) \rho(t, d y)\right| & =\left|\int_{\mathbb{R}} F^{\prime}(M(t, y)) \phi(y) \rho(t, d y)\right| \\
& =\left|\int_{\mathbb{R}}\left(v_{0} \circ N_{0} \circ M(t, y)\right) \phi(y) \rho(t, d y)\right| \\
& \leq \int_{\mathbb{R}}\left|\left(v_{0} \circ N_{0} \circ M(t, y)\right) \phi(y)\right| \rho(t, d y) \\
& \leq\left\|v_{0}\right\|_{L^{\infty}(\mathbb{R})}\|\phi\|_{L^{1}(\rho(t, \cdot))} .
\end{aligned}
$$

Thus, $v(t, \cdot)$ is essentially bounded with respect to $\rho(t, \cdot)$ and $\|v(t, \cdot)\|_{L^{\infty}(\rho(t, \cdot))} \leq\left\|v_{0}\right\|_{L^{\infty}(\mathbb{R})}$.

(4) What we need to show is that for all $\xi \in C_{c}^{\infty}((0, \infty) \times \mathbb{R})$ :

$$
\int_{0}^{\infty} \int_{\mathbb{R}} \partial_{t} \xi(t, y) M(t, y) d y d t-\int_{0}^{\infty} \int_{\mathbb{R}} v(t, y) \xi(t, y) M(t, d y) d t=0 .
$$

So suppose $\xi \in C_{c}^{\infty}((0, \infty) \times \mathbb{R})$. Define $\left.\psi:(0, \infty) \times \mathbb{R}\right) \rightarrow \mathbb{R}$ by:

$$
\psi(t, y):=\int_{-\infty}^{y} \xi(t, z) d z .
$$

Now, by the definition of the distributional derivative of a function, we know for the probability measure $\rho(t, \cdot)$, which is a Radon measure, that for all $\phi \in C_{c}^{\infty}((0, \infty) \times \mathbb{R})$ :

$$
\int_{\mathbb{R}} \phi(t, y) \rho(t, d y)=-\int_{\mathbb{R}} \partial_{y} \phi(t, y) M(t, y) d y .
$$


Then, since $\partial_{y} \psi \in C_{c}^{\infty}((0, \infty) \times \mathbb{R})$, by Lemma 3.3.2, we have that:

$$
\begin{aligned}
0 & =\int_{0}^{\infty} \int_{\mathbb{R}}\left[\partial_{t} \psi(t, y)+v(t, y) \partial_{y} \psi(t, y)\right] \rho(t, d y) d t \\
& =\int_{0}^{\infty} \int_{\mathbb{R}} \partial_{t} \psi(t, y) \rho(t, d y) d t+\int_{0}^{\infty} \int_{\mathbb{R}} v(t, y) \partial_{y} \psi(t, y) \rho(t, d y) d t \\
& =-\int_{0}^{\infty} \int_{\mathbb{R}} \partial_{t} \partial_{y} \psi(t, y) M(t, y) d y d t+\int_{0}^{\infty} \int_{\mathbb{R}} v(t, y) \partial_{y} \psi(t, y) \rho(t, d y) d t \\
& =-\int_{0}^{\infty} \int_{\mathbb{R}} \partial_{t} \xi(t, y) M(t, y) d y d t+\int_{0}^{\infty} \int_{\mathbb{R}} v(t, y) \xi(t, y) \rho(t, d y) d t
\end{aligned}
$$

Thus we have shown what we needed to show.

Thus we have now shown that the result from [6] applies and so the solution characterized by the sticky particle system and the solution characterized by the scalar conservation law are actually one and the same solution $(\rho, v)$. More precisely, Proposition 3.3.2 and Proposition 3.3.3 combine with Corollary 3.3.1 to yield:

Theorem 3.3.2. Let $\rho_{0} \in \mathcal{P}_{2}(\mathbb{R})$ and $v_{0}: \mathbb{R} \rightarrow \mathbb{R}$ be right-continuous and bounded. Then there exists a unique SPF solution to (PE-IC), which coincides with the SCL solution to (PE-IC).

\subsection{Existence of an SPF solution for general initial data}

\subsubsection{More General $v_{0}$}

We now extend the existence result on (PE-IC) in [7] to more general initial conditions $\rho_{0}$ and $v_{0}$. We still consider $\rho_{0}$ in the space of probability measures with finite second moment, i.e. $\rho_{0} \in \mathcal{P}_{2}(\mathbb{R})$, but now we allow $v_{0}$ to be square integrable with respect to $\rho_{0}$ (yet not necessarily absolutely continuous).

We do so by approximating the initial condition $v_{0}$ with initial conditions $v_{0, n}$ from the space of smooth functions of compact support, a space which is dense in the space of bounded, square integrable functions with respect to $\rho_{0}$ and whose members are absolutely continuous. The next proposition shows that if a sequence of SPF solutions converges (assumption (6) in Theorem 3.4.1) to a distributional solution for (PE-IC), then the limiting solution is an SPF solution as well (in particular, it is described by a Lagrangian map $X$ ).

Proposition 3.4.1. Suppose:

1. $\rho_{0} \in \mathcal{P}_{2}(\mathbb{R})$,

2. $v_{0} \in L^{2}\left(\rho_{0}\right)$, i.e. $v_{0}: \mathbb{R} \rightarrow \mathbb{R}, v_{0}$ is measurable, and $\int_{\mathbb{R}} v_{0}^{2} d \rho_{0}<\infty$,

3. $\left\{v_{0, n}\right\}_{n} \subset L^{2}\left(\rho_{0}\right)$ is a sequence converging to $v_{0}$ in $L^{2}\left(\rho_{0}\right)$,

4. For each $n, X_{n}:[0, \infty) \rightarrow L^{2}\left(\rho_{0}\right)$ is a solution to SPF-IC with $\rho_{0}$ and $v_{0}=v_{0, n}$ (note that in ["]] it was shown that $X_{n}(t)$ is non-decreasing), 
5. For each $n$ set $\rho_{n}(t, \cdot)=X_{n}(t, \cdot)_{\#} \rho_{0}$, and $v_{n}\left(t, X_{n}(t, \cdot)\right)=\mathbb{E}_{\rho_{0}}\left[v_{0, n} \mid X_{n}(t, \cdot)\right]$.

6. For each $t \in[0, \infty), \rho(t, \cdot) \in \mathcal{P}_{2}(\mathbb{R})$ is such that $\rho_{n}(t, \cdot) \underset{n \rightarrow \infty}{\longrightarrow} \rho(t, \cdot)$ in the Wasserstein distance, $v(t, \cdot) \in L^{2}\left(\rho_{0}\right)$ is such that for all $\phi \in C_{b}(\mathbb{R})$

$$
\int_{\mathbb{R}} v_{n}(t, y) \phi(y) \rho_{n}(t, d y) \underset{n \rightarrow \infty}{\longrightarrow} \int_{\mathbb{R}} v(t, y) \phi(y) \rho(t, d y)
$$

and $(\rho, v)$ is the solution to $(\mathrm{PE})$ for initial conditions $\left(\rho_{0}, v_{0}\right)$.

Then:

1. For each $t \in[0, \infty)$, there exists $X(t, \cdot) \in L^{2}\left(\rho_{0}\right)$ such that $X_{n}(t, \cdot) \underset{n \rightarrow \infty}{\longrightarrow} X(t, \cdot)$.

2. This $X$ satisfies (SPF-IC).

Proof. : (1) Assume $t \in[0, \infty)$. Now examine the sequence $\left\{X_{n}(t, \cdot)\right\}_{n}$ of functions in $L^{2}\left(\rho_{0}\right)$. Note that since $X_{n}(t, \cdot)$ is non-decreasing, it is the optimal map pushing $\rho_{0}$ to $\rho_{n}(t, \cdot)$. Let $\lambda=\left.\mathcal{L}\right|_{[0,1]}$ be the Lebesgue measure on $[0,1]$. Optimal transport theory guarantees there exists a unique non-decreasing map pushing $\left.\mathcal{L}\right|_{[0,1]}$ to $\rho_{0}$, call it $N_{0}$, and a unique non-decreasing map pushing $\left.\mathcal{L}\right|_{[0,1]}$ to $\rho_{n}(t, \cdot)$, call it $N_{n}(t, \cdot)$. Since $N_{0}$ is non-decreasing and $X_{n}(t, \cdot)$ is nondecreasing, $X_{n}(t, \cdot) \circ N_{0}$ is non-decreasing. Thus $X_{n}(t, \cdot) \circ N_{0}$ is the unique non-decreasing map pushing $\left.\mathcal{L}\right|_{[0,1]}$ to $\rho_{n}(t, \cdot)$. But so is $N_{n}(t, \cdot)$, and thus $X_{n}(t, \cdot) \circ N_{0}=N_{n}(t, \cdot)$. Thus, for given $n, m$,

$$
\begin{aligned}
\left\|X_{n}(t, \cdot)-X_{m}(t, \cdot)\right\|_{L^{2}\left(\rho_{0}\right)}^{2} & =\int_{0}^{1}\left|X_{n}\left(t, N_{0}(x)\right)-X_{m}\left(t, N_{0}(x)\right)\right|^{2} d x \\
& =\int_{0}^{1}\left|N_{n}(t, x)-N_{m}(t, x)\right|^{2} d x \\
& =W_{2}^{2}\left(\rho_{n}(t, \cdot), \rho_{m}(t, \cdot)\right) .
\end{aligned}
$$

From the assumptions, $\rho_{n}(t, \cdot) \underset{n \rightarrow \infty}{\longrightarrow} \rho(t, \cdot)$ in the Wasserstein distance, thus $\left\{\rho_{n}(t, \cdot)\right\}_{n}$ is Cauchy. Therefore, $\left\{X_{n}(t, \cdot)\right\}_{n}$ is Cauchy, so there exists $X(t, \cdot) \in L^{2}\left(\rho_{0}\right)$ such that $X_{n}(t, \cdot) \underset{n \rightarrow \infty}{\longrightarrow} X(t, \cdot)$. Now we know that for all $\phi \in C_{b}(\mathbb{R})$,

$$
\int_{\mathbb{R}} \phi\left(X_{n}(t, y)\right) \rho_{0}(d y)=\int_{\mathbb{R}} \phi(y) \rho_{n}(t, d y) .
$$

Since $\phi$ is continuous and bounded, and $X_{n}(t, \cdot)$ converges strongly to $X(t, \cdot)$ in $L^{2}\left(\rho_{0}\right)$, by the dominated convergence theorem we have, for all $\phi \in C_{b}(\mathbb{R})$,

$$
\int_{\mathbb{R}} \phi\left(X_{n}(t, y)\right) \rho_{0}(d y) \rightarrow \int_{\mathbb{R}} \phi(X(t, y)) \rho_{0}(d y) .
$$

But the convergence in the Wasserstein distance implies, for all $\phi \in C_{b}(\mathbb{R})$,

$$
\int_{\mathbb{R}} \phi(y) \rho_{n}(t, d y) \rightarrow \int_{\mathbb{R}} \phi(y) \rho(t, d y)
$$


and so, for all $\phi \in C_{b}(\mathbb{R})$, we have

$$
\int_{\mathbb{R}} \phi(X(t, y)) \rho_{0}(d y)=\int_{\mathbb{R}} \phi(y) \rho(t, d y),
$$

i.e. $X(t, \cdot)_{\#} \rho_{0}=\rho_{t}$.

(2) Again, the optimal transport theory guarantees there exists a unique non-decreasing map pushing $\lambda:=\left.\mathcal{L}\right|_{[0,1]}$ to $\rho(t, \cdot)$, call it $\left.N(t, \cdot)\right)$. Since all $X_{n}(t, \cdot)$ are non-decreasing and $X_{n}(t, \cdot) \underset{n \rightarrow \infty}{\longrightarrow} X(t, \cdot), X(t, \cdot)$ is non-decreasing. Thus $X(t, \cdot) \circ N_{0}$ is non-decreasing. So $X(t, \cdot) \circ N_{0}$ is also the unique non-decreasing map pushing $\left.\mathcal{L}\right|_{[0,1]}$ forward to $\rho(t, \cdot)$, and thus $X(t, \cdot) \circ N_{0}=N(t, \cdot)$. Now $N \in W^{1,1}\left(0, T ; L^{1}(0,1)\right)$, thus from [12], there is a $v$ such that $v$ is the integrable velocity of $\rho$ and:

$$
\dot{N}(t, x)=v(t, N(t, x)) \text { for Lebesgue a.e. } x \in[0,1] .
$$

In other words

$$
N(t, x)=N_{0}(x)+\int_{0}^{t} v(\tau, N(\tau, x)) d \tau \text { for Lebesgue a.e. } x \in[0,1] .
$$

Thus, since $X(t, \cdot) \circ N_{0}=N(t, \cdot)$, we have

$$
X(t, \cdot) \circ N_{0}(x)=N_{0}(x)+\int_{0}^{t} v(\tau, X(\tau, \cdot)) \circ N_{0}(x) d \tau \text { for Lebesgue a.e. } x \in[0,1] .
$$

Letting $y=N_{0}(x)$, we see that, because $N_{0 \#} \lambda=\rho_{0}$ :

$$
X(t, y)=y+\int_{0}^{t} v(\tau, X(\tau, y)) d \tau \text { for } \rho_{t} \text { a.e. } y \in \mathbb{R} .
$$

On the other hand, we have

$$
\int_{\mathbb{R}} v_{n}(t, y) \phi(y) \rho_{n}(t, d y)=\int_{\mathbb{R}} v_{0}(y) \phi\left(X_{n}(t, y)\right) \rho_{0}(d y) \text { for all } \phi \in C_{b}(\mathbb{R}),
$$

so the convergence of $X_{n}(t, \cdot)$ to $X(t, \cdot)$ in $L^{2}\left(\rho_{0}\right)$ implies, along with the hypothesis on the narrow convergence of $v_{n} \rho_{n}$ to $v \rho$, that we can pass to the limit in the displayed inequality above to obtain $v(t, X(t, \cdot))=\mathbb{E}_{\rho_{0}}\left[v_{0} \mid X(t, \cdot)\right]$. It follows, in view of (3.4.1), that

$$
\dot{X}(t, \cdot)=v(t, X(t, \cdot))=\mathbb{E}_{\rho_{0}}\left[v_{0} \mid X(t, \cdot)\right] \text { for all } t \in[0, \infty) .
$$

Now we know that $X_{n}(0, y)=y$ for $\rho_{0}$ a.e. $y$, thus for each $n$ there exists $A_{n} \subset \mathbb{R}$ such that $\rho_{0}\left(A_{n}\right)=0$ and $X_{n}(0, y)=y$ for all $y \in \mathbb{R} \backslash A_{n}$. Since $X_{n}(0, \cdot) \underset{n \rightarrow \infty}{\longrightarrow} X(0, \cdot)$, we see that $X(0, y)=y$ for at least all $y \in \mathbb{R} \backslash\left(\cup_{i=1}^{\infty} A_{n}\right)$. Now $\rho_{0}\left(\cup_{i=1}^{\infty} A_{n}\right)=0$, thus $X(0, y)=y$ for $\rho_{0}$-a.e. $y$, i.e. $X(0, \cdot)=\operatorname{Id}_{\mathbb{R}}, \rho_{0}$ a.e. Therefore $X$ satisfies (SPF-IC).

Finally, we are now in a position to extend the existence result from [7] to any squareintegrable velocity. 
Theorem 3.4.1. Let $\rho_{0} \in \mathcal{P}_{2}(\mathbb{R})$ and $v_{0} \in L^{2}\left(\rho_{0}\right)$. Then there exists an SPF solution to $(P E-I C)$.

Proof. Let $\left\{v_{0, n}\right\}_{n} \subset C_{c}(\mathbb{R})$ be a sequence which converges to $v_{0}$ in $L^{2}\left(\rho_{0}\right)$. Consider, for each $n \geq 1$, an SPF solution as in Theorem 3.1.1 (existence proved in [7]). According to Theorem 3.3.2, this SPF solution is unique, as it coincides with the SCL solution stemming from the same initial data $\left(\rho_{0}, v_{0, n}\right)$. By Corollary 3.2.1 and Lemma 3.2.1 we deduce that, upon denoting by $(\rho, v)$ the SCL solution corresponding to $\left(\rho_{0}, v_{0}\right)$, the solutions $\left(\rho_{n}, v_{n}\right)$ and $(\rho, v)$ satisfy the hypotheses of Proposition 3.4.1. Thus, Proposition 3.4.1 yields the existence of a Lagrangian map $X$ which satisfies (SPF-IC) and $X(t, \cdot)_{\#} \rho_{0}=\rho(t, \cdot)$ for all $t \geq 0$. 


\section{Chapter 4}

\section{Open Problems}

\subsection{Boundary conditions}

Recently we have begun working on the sticky particle system problem in the presence of boundary conditions. In this formulation, there are two walls located at $x=x_{0}$ and $x=x_{1}$. We assume, without loss of generality, that the initial mass distribution is supported on the interval $\left[x_{0}, x_{1}\right]$.

For the conservation equations and sticky particles, the boundary is typically modelled as an elastic collision with an infinite mass particle, which then leads to the angle of reflection of a sticky particle being equal to the angle of incidence of the sticky particle.

Several scenarios with a finite number of sticky particles were investigated in order to begin gaining insight into the expected behavior. The first scenario shown below is for 10 particles, all of equal mass and initially equally spaced between 0 and 10 and all with initial velocity of -1 . 


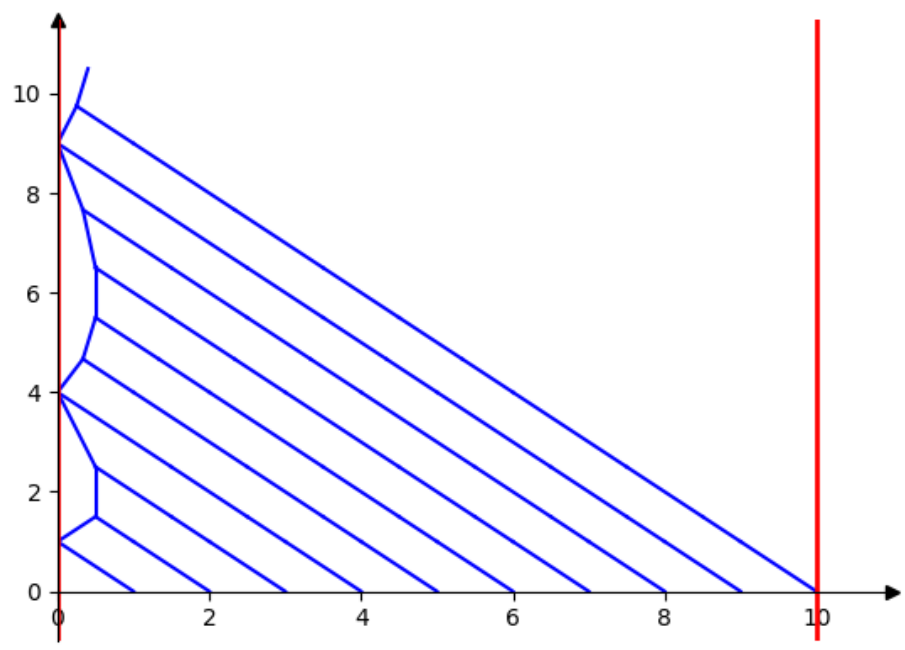

Figure 4.1: 10 Sticky Particles with Boundary

It can be seen that as the system evolves, the leftmost particle increases in mass; thus requiring interactions with more and more particles from the right in order to force the combined particle to have a negative velocity.

A second scenario shown below is for 30 particles, all of equal mass and initially spaced between 0 and 30 and all with initial velocity of -1 . 


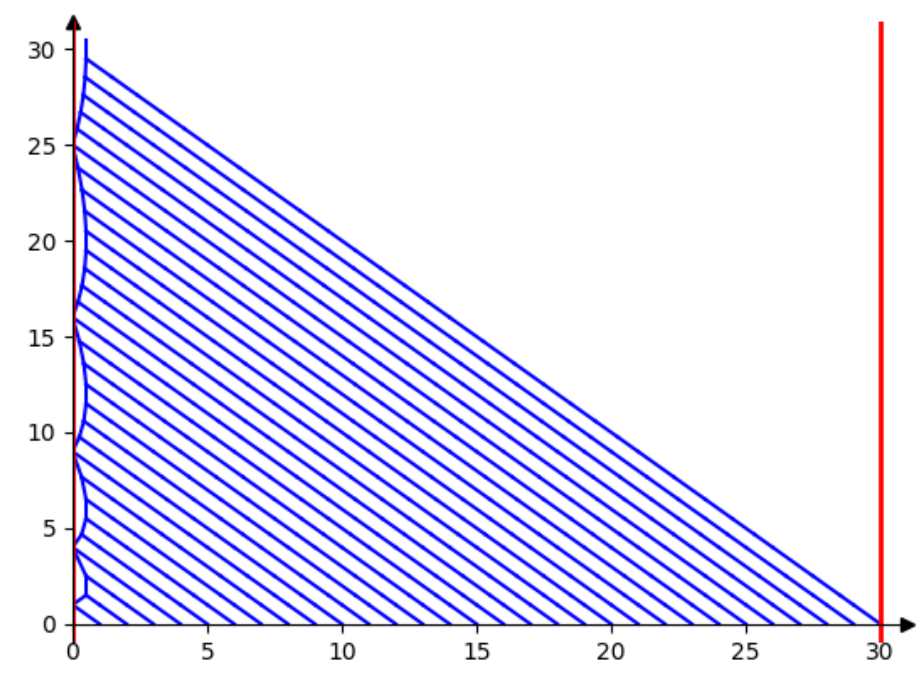

Figure 4.2: 30 Sticky Particles with Boundary

The pattern seen with 10 particles continues in this scenario of 30 particles. However, this is merely a few finite sticky particle cases; further research is needed in order to develop generally applicable results for probability distribution masses.

\subsection{Unique Eulerian Solution}

Note that in the uniqueness result for $v_{0} \in L^{\infty}\left(\rho_{0}\right)$, we relied on the Lagrangian solution to extend the Oleinik result to an everywhere Oleinik result. It is an open problem as to whether there is a corresponding uniqueness result for the Eulerian solution. 


\section{Chapter 5}

\section{Appendices}

\subsection{Examples}

Let $\rho_{0}=\delta_{0}$ and $v_{0}=0$.

1. The stationary solution (to (PE-IC)) $\left(\rho_{0}, v_{0}\right)$ satisfies both (SICE) and (e-Oleinik). So, this is the SPF/SCL solution; also the sticky particles solution. The Lagrangian map is $X(t, \cdot)=0$ for all $t$.

2. One can easily check that if

$$
\rho_{t}:=\frac{1}{2}\left(\delta_{-t}+\delta_{t}\right) \text { and } v_{t}(-t)=-1, v_{t}(t)=1,
$$

then $(\rho, v)$ solves (PE-IC). It turns out that $[0, T] \ni t \rightarrow \rho_{t}$ is the geodesic in the Wasserstein space $\mathcal{P}_{2}(\mathbb{R})$ connecting $\rho_{0}$ and $\rho_{T}$ for any $T>0$. Note that $(\rho, v)$ satisfies (e-Oleinik) but does not satisfy (SICE).

3. For all integers $k \geq 0$ define

$$
\alpha_{k}:\left[2^{-k-1}, 2^{-k}\right) \rightarrow \mathbb{R}, \quad \alpha_{k}(t)=3 \cdot 2^{-k-1} t-2^{-1} 4^{-k}
$$

and let

$$
\bar{\rho}_{t}:=\frac{1}{2}\left[\delta_{-\alpha_{k}(t)}+\delta_{\alpha_{k}(t)}\right] \text { if } t \in\left[2^{-k-1}, 2^{-k}\right) .
$$

For $t \geq 1$ we take

$$
\bar{\rho}_{t}:=\frac{1}{2}\left(\delta_{-t}+\delta_{t}\right)
$$

If we set

$$
\bar{v}_{t}\left(-\alpha_{k}(t)\right):=-3 \cdot 2^{-k-1}, \quad \bar{v}_{t}\left(\alpha_{k}(t)\right):=3 \cdot 2^{-k-1} \text { for } t \in\left[2^{-k-1}, 2^{-k}\right)
$$

and

$$
\bar{v}_{t}(-t)=-1, \quad v_{t}(t)=1 \text { for } t \geq 1,
$$

we can check that $(\bar{\rho}, \bar{v})$ also satisfies $(\mathrm{PE})$ with the same initial conditions $\left(\bar{\rho}_{0}=\delta_{0}, \bar{v}_{0}=0\right)$. However, it is not hard to see that while $(\bar{\rho}, \bar{v})$ satisfies (SICE), it does not satisfy (Oleinik) at any time $t>0$. 


\subsection{Borel Sets and Maps}

Proposition 5.2.1. Suppose $\rho \in A C_{\text {loc }}^{2}\left([0, \infty) ; \mathcal{P}_{2}(\mathbb{R})\right)$, i.e. there exists a Borel map $v$ : $[0, \infty) \times \mathbb{R} \rightarrow \mathbb{R}$ such that $(\rho, v)$ satisfies

$$
\partial_{t} \rho+\partial_{y}(\rho v)=0 \text { in the sense of distributions on }[0, \infty) \times \mathbb{R}
$$

and

$$
\text { for any } 0<T<\infty \text { we have } \int_{0}^{T}\|v(t, \cdot)\|_{L^{2}(\rho(t, \cdot))}^{2} d t=: C(T)<\infty .
$$

Let $M(t, \cdot)$ be the right continuous cumulative distribution function of $\rho(t, \cdot)$. Let $N(t, \cdot)$ be the right continuous optimal map pushing $\left.\mathcal{L}^{1}\right|_{I}$ forward to $\rho(t, \cdot)$, where $I:=(0,1)$. Then the following maps are Borel:

1. $M(t, y):=\rho(t,(-\infty, y])$ on $[0, \infty) \times \mathbb{R}$;

2. $l(t, y):=\sup \{z \in \mathbb{R}: M(t, z)<M(t, y)\}$ on $([0, \infty) \times \mathbb{R}) \backslash(\mathcal{O} \cup \mathcal{Z})$, where $\mathcal{O}:=\{(t, y) \in$ $[0, \infty) \times \mathbb{R}: M(t, y)=1\}, \mathcal{Z}:=\{(t, y) \in[0, \infty) \times \mathbb{R}: M(t, y)=0\}$

3. $r(t, y):=\inf \{z \in \mathbb{R}: M(t, z)>M(t, y)\}$ on $([0, \infty) \times \mathbb{R}) \backslash(\mathcal{O} \cup \mathcal{Z})$.

Proof. : (1) Note that $M(t,-\infty)=0, M(t, \infty)=1$, and $M(t, \cdot)$ is nondecreasing and rightcontinuous for all $t \in[0, \infty)$. To unburden notation, we denote $\rho_{t}:=\rho(t, \cdot)$ and $v_{t}:=v(t, \cdot)$.

Let $\eta^{\epsilon}$ be the standard mollifier supported in $(-\epsilon, \epsilon)$, shifted to the left by $\epsilon$ (thus it is really $\eta^{\epsilon}(z+\epsilon)$, but we call it $\eta^{\epsilon}$ for notational ease). In [9] it is noted that if we define $M^{\epsilon}(t, y)=\eta^{\epsilon} * M_{t}(y)=\int_{\mathbb{R}} \eta^{\epsilon}(z-y) M(t, z) d z$ (so we mollify in space only), then $M^{\epsilon}(t, y) \underset{\epsilon \rightarrow 0^{+}}{\longrightarrow}$ $M(t, y)$ pointwise for all $(t, y) \in[0, \infty) \times \mathbb{R}$, due to the right continuity of $M(t, \cdot)$. So it is enough to show $M^{\epsilon}$ is continuous (jointly in $(t, y)$ ) to conclude that $M$ is jointly Borel. Define $\Theta^{\epsilon}(z):=\int_{-\infty}^{z} \eta^{\epsilon}(s) d s$ and note that $\Theta^{\epsilon}(-\infty)=0$ and $\Theta^{\epsilon}(\infty)=1$. In addition, note that since $\eta^{\epsilon} \geq 0, \Theta^{\epsilon}$ is nondecreasing on $\mathbb{R}$ (it is a cumulative distribution, just like $M(t, \cdot)$ ). Suppose $(t, y),(s, x) \in[0, \infty) \times \mathbb{R}$. Then

$$
\begin{aligned}
M^{\epsilon} & (t, y)-M^{\epsilon}(s, x)=\int_{\mathbb{R}} \eta^{\epsilon}(z-y) M(t, z) d z-\int_{\mathbb{R}} \eta^{\epsilon}(z-x) M(s, z) d z \\
& =\int_{\mathbb{R}} \frac{d}{d z}\left[\Theta^{\epsilon}(z-y)\right] M(t, z) d z-\int_{\mathbb{R}} \frac{d}{d z}\left[\Theta^{\epsilon}(z-x)\right] M(s, z) d z \\
& =\left.\Theta^{\epsilon}(z-y) M(t, z)\right|_{-\infty} ^{\infty}-\left.\Theta^{\epsilon}(z-x) M(s, z)\right|_{-\infty} ^{\infty} \\
& -\int_{\mathbb{R}} \Theta^{\epsilon}(z-y) \rho_{t}(d z)+\int_{\mathbb{R}} \Theta^{\epsilon}(z-x) \rho_{s}(d z) \\
= & -\int_{\mathbb{R}} \Theta^{\epsilon}(z-y) \rho_{t}(d z)+\int_{\mathbb{R}} \Theta^{\epsilon}(z-x) \rho_{s}(d z) \\
= & \int_{\mathbb{R}}\left[\Theta^{\epsilon}(z-x)-\Theta^{\epsilon}(z-y)\right] \rho_{t}(d z)+\left[\int_{\mathbb{R}} \Theta^{\epsilon}(z-x) \rho_{s}(d z)-\int_{\mathbb{R}} \Theta^{\epsilon}(z-x) \rho_{t}(d z)\right] \\
= & \int_{\mathbb{R}}(y-x) \eta^{\epsilon}(\alpha(z, x, y)) \rho_{t}(d z)+\int_{s}^{t} \int_{\mathbb{R}} \eta^{\epsilon}(z-x) v_{\tau}(z) \rho_{\tau}(d z) d \tau .
\end{aligned}
$$


In the last line, we used the Mean Value Theorem for $\Theta^{\epsilon}$ for the first term and

$$
\frac{d}{d t} \int_{\mathbb{R}} \phi(z) \rho_{t}(d z)=\int_{\mathbb{R}} \phi^{\prime}(z) v_{t}(z) \rho_{t}(d z) \text { for all } \phi \in C_{c}^{1}(\mathbb{R})
$$

for the second. Thus, by (5.2.1),

$$
\begin{aligned}
\left|M^{\epsilon}(t, y)-M^{\epsilon}(s, x)\right| & \leq|y-x| \max \eta^{\epsilon}+\int_{s}^{t} \int_{\mathbb{R}} \eta^{\epsilon}(z-x)\left|v_{\tau}(z)\right| \rho_{\tau}(d z) d \tau \\
& \leq|y-x| \max \eta^{\epsilon}+|t-s|^{1 / 2}\left(\max \eta^{\epsilon}\right)[C(T)]^{1 / 2}
\end{aligned}
$$

so $M^{\epsilon}$ is (jointly) continuous on $[0, T] \times \mathbb{R}$, which implies $M$ is jointly Borel.

(3) By (1), $\mathcal{O}$ and $\mathcal{Z}$ are Borel sets. If $0<x<1$, we have $\inf \{z \in \mathbb{R}: M(t, z)>x\}=N(t, x)$, so $r(t, y)=N(t, M(t, y))$ if $(t, y) \in([0, \infty) \times \mathbb{R}) \backslash(\mathcal{O} \cup \mathcal{Z})$. We just showed that $M$ is Borel, so it is enough to show that $N$ is Borel in order to conclude that $r$ is Borel on the Borel set $([0, \infty) \times \mathbb{R}) \backslash(\mathcal{O} \cup \mathcal{Z})$

Claim: $N$ is Borel on $[0, \infty) \times(0,1)$. Proof: Let $0<T<\infty$ and $0<\delta<\frac{1}{2}$ and consider the restriction of $N$ to $[0, T] \times[\delta, 1-\delta]$. Use the same mollifier $\eta^{\epsilon}$ (as in the proof above that $M$ is Borel); since $N(t, \cdot)$ is right continuous, we have $\eta^{\epsilon} * N(t, \cdot) \underset{\epsilon \rightarrow 0^{+}}{\longrightarrow} N(t, \cdot)$ pointwise everywhere on $[\delta, 1-\delta]$. So let $N^{\epsilon}(t, x)=\left[\eta^{\epsilon} * N(t, \cdot)\right](x)$. Since $N^{\epsilon} \underset{\epsilon \rightarrow 0^{+}}{\stackrel{\epsilon \rightarrow 0^{+}}{\longrightarrow}} N$ everywhere on $[0, T] \times[\delta, 1-\delta]$, it is enough to show that $N^{\epsilon}$ is jointly continuous in order to conclude that $N$ is Borel on $[0, T] \times[\delta, 1-\delta]$. So let $s, t \in[0, T]$ and $x_{1} \leq x_{2} \in[\delta, 1-\delta]$ to estimate (for all sufficiently small $\epsilon>0$ )

$$
\begin{aligned}
\left|N^{\epsilon}\left(s, x_{1}\right)-N^{\epsilon}\left(t, x_{2}\right)\right| & \leq\left|N^{\epsilon}\left(s, x_{1}\right)-N^{\epsilon}\left(t, x_{1}\right)\right|+\left|N^{\epsilon}\left(t, x_{1}\right)-N^{\epsilon}\left(t, x_{2}\right)\right| \\
& \leq \int_{x_{1}-2 \epsilon}^{x_{1}} \eta^{\epsilon}\left(x_{1}-z\right)|N(s, z)-N(t, z)| d z \\
& +\int_{\frac{\delta}{2}}^{1-\delta}\left|\eta^{\epsilon}\left(x_{1}-z\right)-\eta^{\epsilon}\left(x_{2}-z\right)\right||N(t, z)| d z \\
& \leq\left(\max \eta^{\epsilon}\right) W_{2}\left(\rho_{s}, \rho_{t}\right)+\left|x_{1}-x_{2}\right| \max \left|\left(\eta^{\epsilon}\right)^{\prime}\right|\|N(t, \cdot)\|_{L^{2}(I)} .
\end{aligned}
$$

But it is known (see, e.g., [12]) that

$$
N(t, z)=N(s, z)+\int_{s}^{t} v_{\tau}(N(\tau, z)) d \tau \text { for Lebesgue a.e. } z \in(0,1) \text { and all } 0 \leq s<t \leq T,
$$

which implies (by (5.2.1))

$$
W_{2}\left(\rho_{s}, \rho_{t}\right)=\|N(s, \cdot)-N(t, \cdot)\|_{L^{2}(I)} \leq|t-s|^{1 / 2}[C(T)]^{1 / 2}
$$

and

$$
\|N(t, \cdot)\|_{L^{2}(I)} \leq\left\|N_{0}\right\|_{L^{2}(I)}+\int_{0}^{T}\left\|v_{\tau}(N(\tau, \cdot))\right\|_{L^{2}(I)} d \tau=\left\|N_{0}\right\|_{L^{2}(I)}+\int_{0}^{T}\left\|v_{\tau}\right\|_{L^{2}\left(\rho_{\tau}\right)} d \tau .
$$

But (5.2.1) also ensures that the last term from above is bounded by $[T C(T)]^{1 / 2}$. Thus, $N^{\epsilon}$ is continuous on $[0, T] \times[\delta, 1-\delta]$, which implies $N$ is Borel on $[0, T] \times[\delta, 1-\delta]$. Since $0<\delta<\frac{1}{2}$ and $T>0$ are arbitrary, $N$ is Borel on $[0, \infty) \times(0,1)$.

(2) We have $l(t, y)=N(t, M(t, y)-)=\lim _{n \rightarrow \infty} N(t, M(t, y)-1 / n)$. But we proved above that both $N$ and $M$ are Borel maps, so $l$ is Borel. 

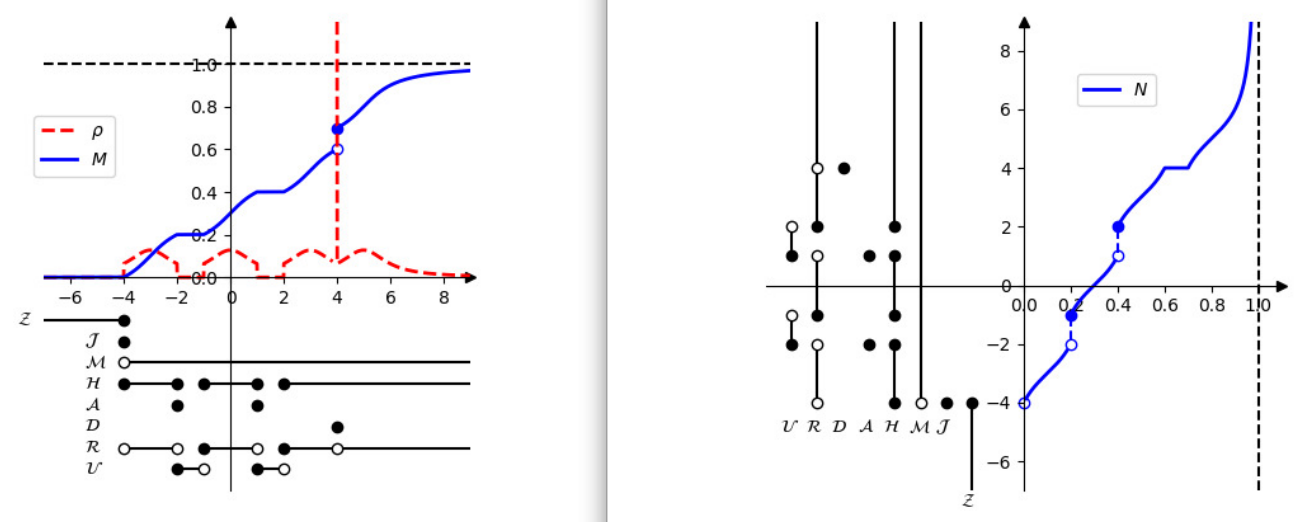

Figure 5.1: An illustration of a CDF $M$ and its generalized inverse $N$ for a probability measure $\rho$ (with a point mass at $y=4$ ). The time $t$ is frozen and the sets shown are the fibers (spatial projections) of the sets defined in Proposition 5.2.2. Note that the sets $\mathcal{S}$ and $\mathcal{O}$ are empty in this case (as $M$ does not reach the value 1 ).

Proposition 5.2.2. Under the assumptions from Proposition 5.2.1, the following sets are Borel:

1. $\mathcal{Z}:=\{(t, y) \in[0, \infty) \times \mathbb{R}: M(t, y)=0\}$;

2. $\mathcal{J}:=\bigcap_{m \in \mathbb{N}}\left\{(t, y) \in[0, \infty) \times \mathbb{R}: M\left(t, y-\frac{1}{m}\right)=0<M\left(t, y+\frac{1}{m}\right)\right\}$;

3. $\mathcal{S}:=\bigcap_{m \in \mathbb{N}}\left\{(t, y) \in[0, \infty) \times \mathbb{R}: M\left(t, y-\frac{1}{m}\right)<1=M\left(t, y+\frac{1}{m}\right)\right\}$;

4. $\mathcal{O}:=\{(t, y) \in[0, \infty) \times \mathbb{R}: M(t, y)=1\}$

5. $\mathcal{M}:=([0, \infty) \times \mathbb{R}) \backslash(\mathcal{Z} \cup \mathcal{J} \cup \mathcal{S} \cup \mathcal{O})$;

6. $\mathcal{H}:=\bigcap_{m \in \mathbb{N}}\left\{(t, y) \in[0, \infty) \times \mathbb{R}: M\left(t, y-\frac{1}{m}\right)<M\left(t, y+\frac{1}{m}\right)\right\}$;

7. $\mathcal{A}:=\bigcup_{k \in \mathbb{N}} \bigcap_{m \geq k}\left\{(t, y) \in[0, \infty) \times \mathbb{R}: M\left(t, y-\frac{1}{m}\right)<M(t, y)=M\left(t, y+\frac{1}{m}\right)\right\}$;

8. $\mathcal{D}:=\{(t, y) \in[0, \infty) \times \mathbb{R}: \rho(t,\{y\})>0\}$

9. $\mathcal{R}:=(\mathcal{M} \cap \mathcal{H}) \backslash(\mathcal{A} \cup \mathcal{D})$; 
10. $\mathcal{U}:=(\mathcal{M} \backslash \mathcal{H}) \cup[\mathcal{M} \cap(\mathcal{A} \backslash \mathcal{D})]$
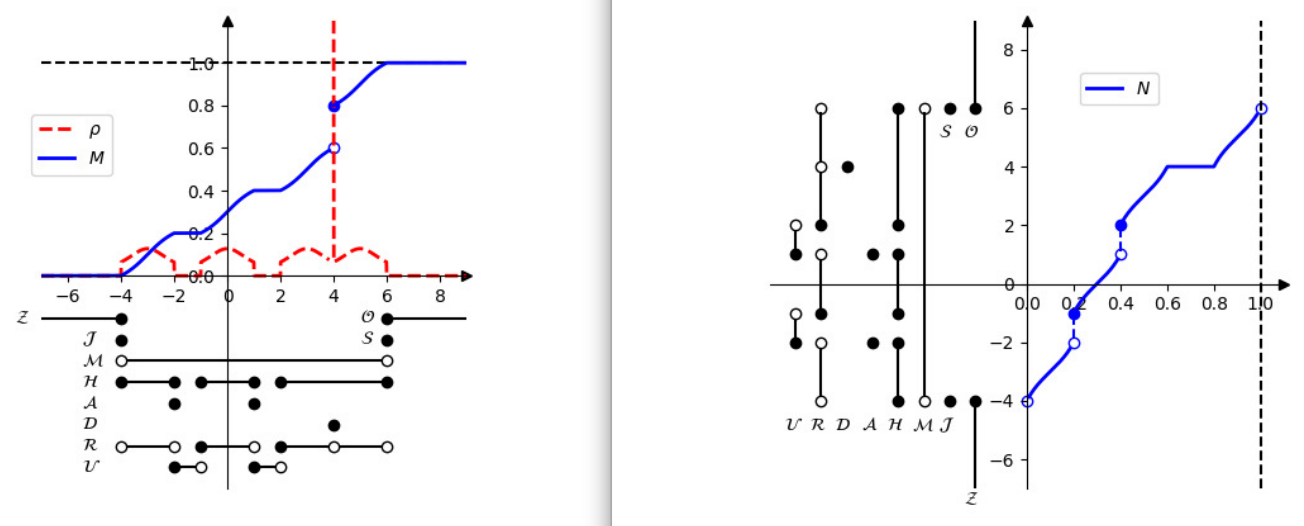

Figure 5.2: Another illustration of a CDF $M$ and its generalized inverse $N$ for a probability measure $\rho$ (with a point mass at $y=4$ ). The time $t$ is frozen and the sets shown are the fibers (spatial projections) of the sets defined in Proposition 5.2.2. Note that in this case the sets $\mathcal{S}$ and $\mathcal{O}$ are non-empty (as $M=1$ on $[6, \infty)$ ).

Except for part (8), all of the above follow directly from Theorem 5.2.1 (1). Part (8) is proved in [4], Lemma 3.5. The purpose of the next result is to elucidate the connections between the sets defined above and the images of various sets of interest through the map $N$.

Proposition 5.2.3. The following properties of the sets defined in the statement of Theorem 5.2.2 hold:

1. $\mathcal{Z} \cup \mathcal{J}=\bigcup_{t \in[0, \infty)}\{t\} \times(-\infty, N(t, 0+)]$, where the closed half-ray $(-\infty, N(t, 0+)]$ is defined as the empty set if $N(t, 0+)=-\infty$;

2. $\mathcal{S} \cup \mathcal{O}=\bigcup_{t \in[0, \infty)}\{t\} \times[N(t, 1-), \infty)$, where the closed half-ray $[N(t, 1-), \infty)$ is defined as the empty set if $N(t, 1-)=\infty$;

3. $\mathcal{M}=\bigcup_{t \in[0, \infty)}\{t\} \times(N(t, 0+), N(t, 1-))$, where the open interval $(N(t, 0+), N(t, 1-))$ is defined as the empty set if $N(t, \cdot)$ is constant;

4. $\mathcal{H}=\bigcup_{t \in[0, \infty)}\{t\} \times \operatorname{spt}\left(\rho_{t}\right)=\bigcup_{t \in[0, \infty)}\{t\} \times[\Lambda \cup N(t, I) \cup N(t, I-)]$, where $N(t, I-):=$ $\{N(t, x-): x \in I\}$ and $\Lambda$ consists of whichever of $N(t, 0+)$ and $N(t, 1-)$ is finite; 
5. $\mathcal{M} \cap(\mathcal{A} \backslash \mathcal{D})=\bigcup_{t \in[0, \infty)}\{t\} \times\{N(t, x-): x \in I$ and $N(t, x-)<N(t, x)\}$;

6. $\mathcal{D}=\bigcup_{t \in[0, \infty)}\{t\} \times\left\{N(t, x): x \in I\right.$ and $\left.[N(t, \cdot)]^{-1}(N(t, x)) \neq\{x\}\right\}$

7. $\mathcal{R}=\bigcup_{t \in[0, \infty)}\{t\} \times\left\{N(t, x): x \in I\right.$ and $\left.[N(t, \cdot)]^{-1}(N(t, x))=\{x\}\right\}$;

8. $\mathcal{U}=\bigcup_{t \in[0, \infty)}\left[\{t\} \times \bigcup_{x \in I}[N(t, x-), N(t, x))\right]$, where the half open interval $[N(t, x-), N(t, x))$ is defined as empty if $N(t, x-)=N(t, x)$. 


\section{Bibliography}

[1] François Bouchut and François James. Duality solutions for pressureless gases, monotone scalar conservation laws, and uniqueness. Communications in Partial Differential Equations, 24(11-12):2173-2189, 1999.

[2] Y. Brenier and E. Grenier. Sticky particles and scalar conservation laws. SIAM J. Numer. Anal., 35:2317, 1998.

[3] Weinan E, Yu. G. Rykov, and Ya. G. Sinai. Generalized variational principles, global weak solutions and behavior with random initial data for systems of conservation laws arising in adhesion particle dynamics. Comm. Math. Phys., 177(2):349-380, 1996.

[4] Mikhail Feldman and Adrian Tudorascu. On lagrangian solutions for the semi-geostrophic system with singular initial data. SIAM Journal on Mathematical Analysis, 45(3):16161640, 2013.

[5] Wilfrid Gangbo and Adrian Tudorascu. On differentiability in the wasserstein space and well-posedness for hamilton-jacobi equations. Journal de Mathématiques Pures et Appliquées, 125:119-174, 2019.

[6] Feimin Huang and Zhen Wang. Well posedness for pressureless flow. Communications in Mathematical Physics, 222(1):117-146, Aug 2001.

[7] Ryan Hynd. Lagrangian coordinates for the sticky particle system. SIAM Journal on Mathematical Analysis, 51(5):3769-3795, 2019.

[8] L. Natile and G. Savaré. A wasserstein approach to the one-dimensional sticky particle system. SIAM J. Math. AnaDevlin2012l., 41:1340, 2009.

[9] Truyen Nguyen and Adrian Tudorascu. Pressureless euler/euler-poisson systems via adhesion dynamics and scalar conservation laws. SIAM Journal on Mathematical Analysis, 40(2):754-775, 2008.

[10] Truyen Nguyen and Adrian Tudorascu. One-dimensional pressureless gas systems with/without viscosity. Communications in Partial Differential Equations, 40(9):1619$1665,2015$.

[11] Walter Rudin. Real and Complex Analysis, 3rd Ed. McGraw-Hill, Inc., USA, 1987.

[12] Adrian Tudorascu. On absolutely continuous curves of probabilities on the line. Discrete and Continuous Dynamical Systems - A, 39(9):5105-5124, 2019. 
[13] Cedric Villani. Topics in Optimal Transportation. American Mathematical Society, 2003.

[14] Y.B. Zeldovich. Gravitational instability: an approximate theory for large density perturbations. Astro. Astrophys., 5:84, 1970. 